\title{
Functional Assessment of Stroke-Induced Regulation of miR-20a-3p and Its Role as a Neuroprotectant
}

\author{
Taylor E. Branyan ${ }^{1,2} \cdot$ Amutha Selvamani $^{1} \cdot$ Min Jung Park ${ }^{1} \cdot$ Kriti E. Korula $^{1} \cdot$ Kelby F. Kosel $^{1} \cdot$ Rahul Srinivasan $^{1,2}$. \\ Farida Sohrabji ${ }^{1,2,3}$
}

Received: 2 May 2021 / Revised: 12 August 2021 / Accepted: 7 September 2021 / Published online: 27 September 2021

(C) The Author(s) 2021

\begin{abstract}
MicroRNAs have gained popularity as a potential treatment for many diseases, including stroke. This study identifies and characterizes a specific member of the miR-17-92 cluster, miR-20a-3p, as a possible stroke therapeutic. A comprehensive microRNA screening showed that miR-20a-3p was significantly upregulated in astrocytes of adult female rats, which typically have better stroke outcomes, while it was profoundly downregulated in astrocytes of middle-aged females and adult and middle-aged males, groups that typically have more severe stroke outcomes. Assays using primary human astrocytes and neurons show that miR-20a-3p treatment alters mitochondrial dynamics in both cell types. To assess whether stroke outcomes could be improved by elevating astrocytic miR-20a-3p, we created a tetracycline (Tet)-induced recombinant adeno-associated virus (rAAV) construct where miR-20a-3p was located downstream a glial fibrillary acidic protein promoter. Treatment with doxycycline induced miR-20-3p expression in astrocytes, reducing mortality and modestly improving sensory motor behavior. A second Tet-induced rAAV construct was created in which miR-20a-3p was located downstream of a neuronspecific enolase (NSE) promoter. These experiments demonstrate that neuronal expression of miR-20a-3p is vastly more neuroprotective than astrocytic expression, with animals receiving the miR-20a-3p vector showing reduced infarction and sensory motor improvement. Intravenous injections, which are a therapeutically tractable treatment route, with miR-20a-3p mimic $4 \mathrm{~h}$ after middle cerebral artery occlusion (MCAo) significantly improved stroke outcomes including infarct volume and sensory motor performance. Improvement was not observed when miR-20a-3p was given immediately or $24 \mathrm{~h}$ after MCAo, identifying a unique delayed therapeutic window. Overall, this study identifies a novel neuroprotective microRNA and characterizes several key pathways by which it can improve stroke outcomes.
\end{abstract}

Keywords Ischemic stroke $\cdot$ MicroRNA $\cdot$ Astrocyte $\cdot$ Blood-brain barrier $\cdot$ Mitochondria $\cdot$ Matrix metalloproteinases

\section{Introduction}

Taylor E. Branyan and Amutha Selvamani have equal contribution.

Farida Sohrabji

f-sohrabji@tamu.edu

1 Women's Health in Neuroscience Program, Neuroscience and Experimental Therapeutics, Texas A\&M Health Science Center College of Medicine, Bryan, TX 77807, USA

2 Texas A\&M Institute for Neuroscience, College Station, TX 77840, USA

3 Department of Neuroscience and Experimental Therapeutics, Texas A\&M Health Science Center College of Medicine, 8447 Riverside Pkwy, Bryan, TX 77807, USA
Stroke remains the leading cause of disability and the fifth leading cause of mortality in the USA. Ischemic stroke accounts for $80 \%$ of all strokes, and there is currently only one FDA-approved drug therapy, tissue plasminogen activator (tPA). tPA must be administered within a short time window of 3-4.5 h to be effective, which limits the proportion of patients eligible to receive this treatment (5.9-7.0\%) [1, 2]. Therefore, there is an urgent need for the development of effective and safe therapeutics. Moreover, since stroke risk and stroke severity are modified by the age and biological sex of the patient, it is imperative for preclinical studies of potential stroke therapies to address both variables.

Epigenetic modifiers, such as small non-coding RNAs, have emerged as powerful candidates for several diseases 
including cancers and immune, infectious, cardiovascular, and neurodegenerative diseases [3-6]. MicroRNAs (miRNAs) are typically 18-25 nucleotides long and bind to complementary sequences in the $3^{\prime}$ UTR of multiple target mRNAs to regulate gene expression [7]. In the case of ischemic stroke, several studies have now shown that miRNA mimics or antagomirs can regulate acute and chronic stroke outcomes [8-12]. Previous work from our lab has shown that the efficacy of miRNA treatment may be restricted to specific age and sex groups. For example, anti-Let7f treatment after middle cerebral artery occlusion (MCAo) improved stroke outcomes in adult female rats but not older females or adult males [8, 13]. Similarly, miR363-3p, identified by miRNA profiling of serum, modifies stroke outcomes only in females (adult and middle-aged) but not age-matched males [14]. In order to identify a more universally effective microRNA treatment, the present study used a novel approach by focusing on astrocytes.

Astrocytes are a crucial cell type for mediating brain energy homeostasis, providing neurotrophic support, and maintaining the blood-brain barrier [15]. Astrocytes have been shown to develop an "aging" phenotype, characterized by increased glial fibrillary acidic protein (GFAP) expression [16] and increased production of senescence-associated secretory phenotype (SASP) factors, such as interleukins (IL-1 $\alpha$ [17], IL-6 [18, 19], IL-8 [20], IL-15 [21]) and matrix metalloproteases (MMP-1 [18], MMP-3, MMP-10 [20]). Astrocytes harvested from the ischemic cortex and striatum in middle-aged females show similar senescence-related changes, including reduced glutamate reuptake, decreased growth factor release, and increased chemokine release compared to adult females, all indicative of decreased neuroprotective capacity [22]. Furthermore, astrocytes from middle-aged females showed reduced trimethylation of histone $\mathrm{H} 3 \mathrm{~K} 4$, a transcriptional enhancer, as compared to adult females [23], indicating a global reduction in transcription potential, including the transcriptional start site for the miR17a-92a cluster.

To assess the role of astrocytic miRNA in stroke outcomes, we compared miRNA profiles in astrocytes obtained from the ischemic forebrain of adult and middle-aged males and females. These groups were specifically selected because stroke outcomes are more severe in adult males and middle-aged males and females as compared to adult females, where stroke-induced infarction and sensory motor impairment is low. This approach confirmed the suppression of the miR-17-92 cluster observed in ChIP-seq analysis [23] and further identified miR-20a-3p as a uniquely age- and sex-regulated miRNA, whose expression was dramatically elevated in adult females as compared to all other groups. Bioinformatics identified mitochondrial and inflammationassociated genes as targets of miR-20a-3p, and select examples were confirmed in molecular and functional assays.
A viral construct designed to conditionally elevated miR20a-3p in astrocytes partially improved stroke outcomes. Subsequently, intravenous injection of fluorescently labeled miR-20a-3p after stroke was found to be preferentially taken up by neurons. A second viral construct was designed to conditionally express miR-20a-3p in neurons, resulting in robust neuroprotection. Finally, i.v. treatment of miR20a-3p, a more translationally viable route of administration, was found to be neuroprotective in both middle-aged males and females. Together, these data indicate that while miR$20 a-3 p$ is produced by and profoundly alters the function of astrocytes after ischemia, stroke neuroprotection via this microRNA may depend on other cell types such as neurons receiving and utilizing miR-20a-3p.

\section{Results}

\section{Expression of MicroRNA from the miR-17-92 Cluster in Astrocytes from Adult and Middle-Aged Males and Females}

Astrocytes were extracted at $48 \mathrm{~h}$ post stroke from adult and middle-aged females and males using positive selection for GLAST. Based on our previous data showing increased trimethylation of the miR-17-92 cluster in adult females, we analyzed sex and age differences in this cluster by qRTPCR (normalized to U6). Members of this miRNA cluster showed a complex regulation due to age and sex. As shown in Fig. 1a, miR-17-5p, miR-18a-5p, miR-19a, miR-19b, miR20a-5p, and miR-20a-3p displayed a significant age $\times$ sex effects, such that each of these miRNAs was elevated in the adult female as compared to the adult male and middle-aged male or female. In the case of miR-17-3p, there were a main effect of age and a main effect of sex, while in the case of miR-92a-3p, there was a main effect of age, where adult animals had a higher expression of these miRNAs as compared to middle-aged animals. The most remarkable age $\times$ sex regulation was seen in miR-20a-3p expression, which demonstrated $a>240,000$-fold elevation in adult female as compared to middle-aged females. A separate analysis of the entire miRnome panel controlling for false discovery rate (FDR) showed that miR-20a-3p was the only astrocytic miRNA that was significantly elevated by age and sex.

\section{miR-20a-3p Expression in the Circulation, Brain, and Astrocytes in Adult and Middle-Aged Females}

To determine if miR-20a-3p suppression is a tissue-specific response to stroke, miR-20a-3p expression was analyzed using qRT-PCR in serum, whole-brain homogenate, and astrocytes from adult and middle-aged females in sham animals and at 2 days post stroke. While basal expression of 


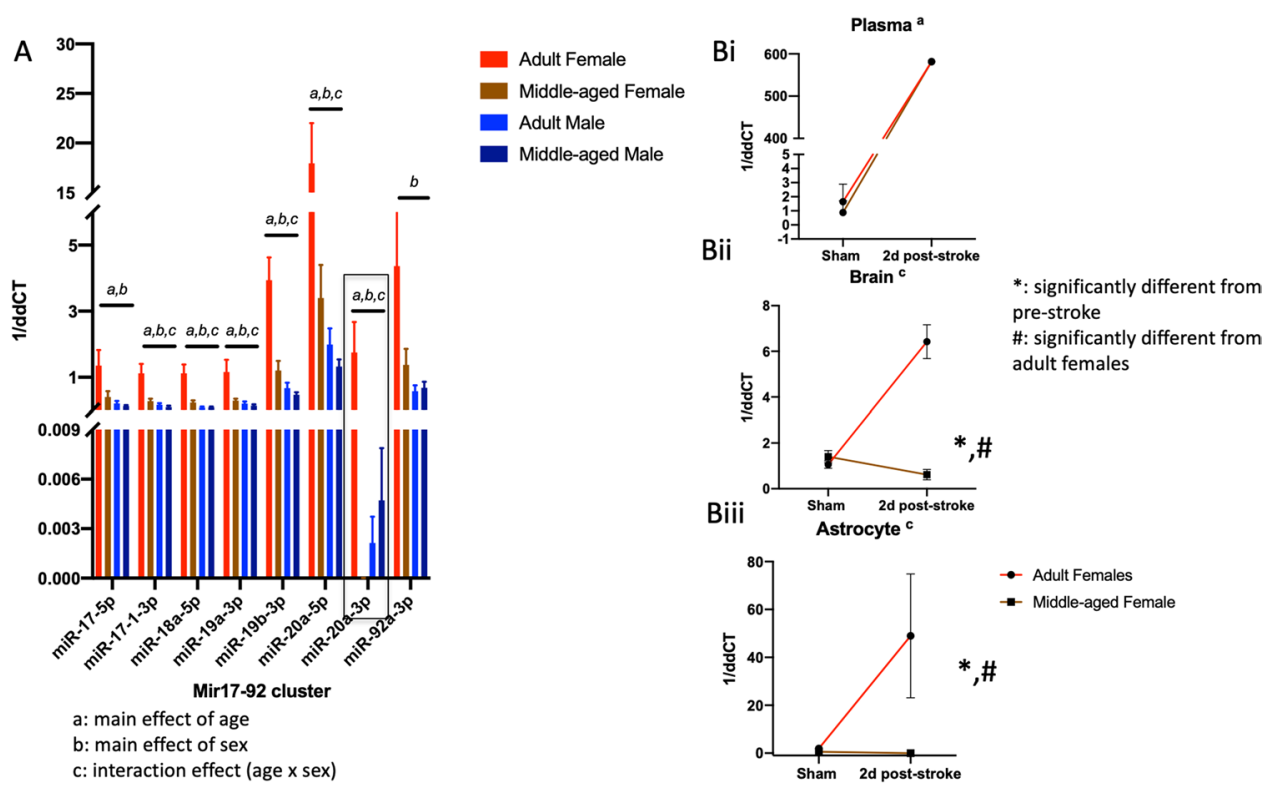

Fig. 1 MiRnome analysis of miR expression from the miR-17-92 cluster. A histogram depicting the mean $( \pm$ SEM $)$ expression of microRNA from the miR-17-92 cluster in adult and middle-aged males and females. Bar above each set indicates main and interaction effects from two-way ANOVAs. $a$ Main effect of age. $b$ Main effect of sex. $c$ Interaction effect (age $\times$ sex). B MiR-20a-3p expression in serum, whole brain, and astrocyte. Pre-stroke and 2-day post-

miR-20a-3p is similar at both ages, miR-20a-3p is altered in an age- and tissue-specific manner (Fig. 1b) during the early acute phase ( 2 days) of stroke. In circulation (serum), miR$20 \mathrm{a}-3 \mathrm{p}$ is significantly elevated at 2 days post stroke in both adult and middle-aged females (Fig. 1b(i)). In whole-brain homogenate (Fig. 1b(ii)) and in astrocytes (Fig. 1b(iii)), miR-20a-3p expression is preferentially elevated in adult females (red line) and profoundly suppressed in middle-aged females (black line) at 2 days post stroke.

\section{Effect of MiR-20a-3p Treatment on Astrocyte Mitochondrial Function In Vitro}

Bioinformatics analyses (using databases TargetScan and MiRWalk 2.0) indicate that miR-20a-3p regulates a large number of genes that are responsible for mediating mitochondrial function (Supplementary Fig. 1). In order to investigate specific mechanisms by which miR-20a-3p alters mitochondrial function, male and female human astrocytes were cultured and subjected to $6 \mathrm{~h}$ of oxygen glucose deprivation (OGD) $\left(1 \% \mathrm{O}_{2}, 0 \mathrm{mM}\right.$ glucose) or normoxia ( $21 \%$ $\mathrm{O}_{2}, 25 \mathrm{mM}$ glucose) and treated with miR-20a-3p mimic, scrambled miR, or vehicle. Astrocyte cultures were confirmed by immunohistochemistry for GFAP (green) and co-labeled with nuclear dye DAPI (blue) (Supplementary Fig. 2a). Cultures that were incubated with $50 \mathrm{nM}$ FAMlabeled miR-20a-3p mimic and MitoTracker Deep Red to stroke serum, brain, and astrocyte samples were evaluated for miR20a expression. Key: asterisk, significantly different from pre stroke; number sign, significantly different from adult females at that time point. $p<0.05$. $n=6$ in most groups, $n=3$ in pre-stroke astrocytes and post stroke brain samples. Red line, adult females; black line, middle-aged females

stain living mitochondria for $6 \mathrm{~h}$ showed that the microRNA is readily taken up by astrocytes (Fig. 2a). In addition, qRT-PCR analysis indicates that female human astrocytes profoundly upregulate miR-20a-3p in response to ischemia, whereas the male astrocytes do not (Fig. 2b), recapitulating our in vivo observations.

To determine the effect of miR-20a-3p on astrocyte survival after OGD, cells were incubated with Calcein-AM dye (Life Technologies, CA) after OGD treatment. MiR-20a-3p treatment significantly increased cell viability in both male and female cells compared to scrambled miR treatment, but with different degrees of efficacy. In females, cell viability in the miR-20a-3p treatment group was virtually similar to that in normoxic controls, while cell viability in males that received miR-20a-3p was greater than that in cultures that received the scrambled oligo but was far lower than that in normoxic controls. Moreover, the female astrocytes demonstrated a greater degree of viability as compared to the male astrocytes after exposure to OGD ( $p=0.002$, Fig. 2c).

Two functional assays were used to explore the effect of miR-20a-3p treatment on astrocytic mitochondrial function. The first technique used was fluorescence recovery after photobleaching (FRAP), in which human astrocyte cultures were labeled with MitoTracker Deep Red and a small area of the cell was photobleached with a high intensity 405-nm laser and the subsequent fluorescent recovery was quantified using ImageJ. MiR-20a-3p treatment significantly increased 
Fig. 2 Effect of miR20a-3p mimic on mitochondrial dynamics. a Primary human astrocytes stained with MitoTracker Deep Red and incubated with FAMlabeled miR-20a-3p for $6 \mathrm{~h}$. b qRT-PCR expression of miR20a-30 in primary human astrocytes. c Calcein assay on female and male human astrocytes. Histogram depicting the mean $( \pm$ SEM) percent of viable cells relative to normoxic conditions. d FRAP analysis of female and male human astrocytes. The recovery was normalized to the level of bleaching in the cells (bleaching value was set at 1). e Seahorse XFe96 Mito Stress Test on human astrocytes. $* p \leq 0.05, * * p \leq 0.01$, $* * * p \leq 0.001, * * * * p \leq 0.0001$
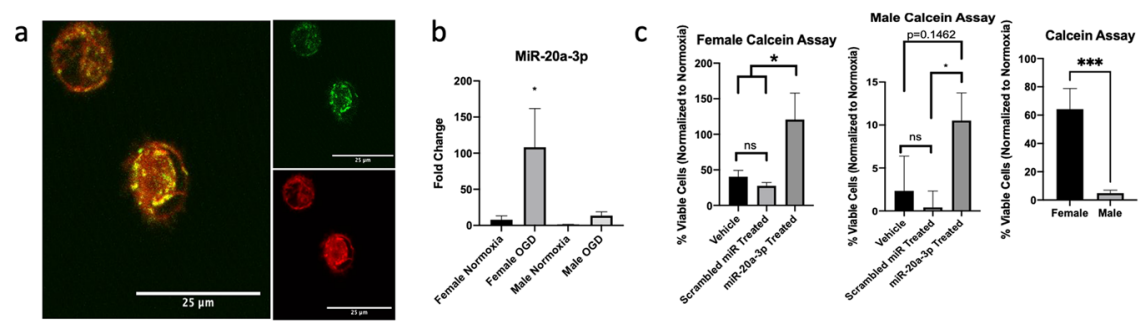

d
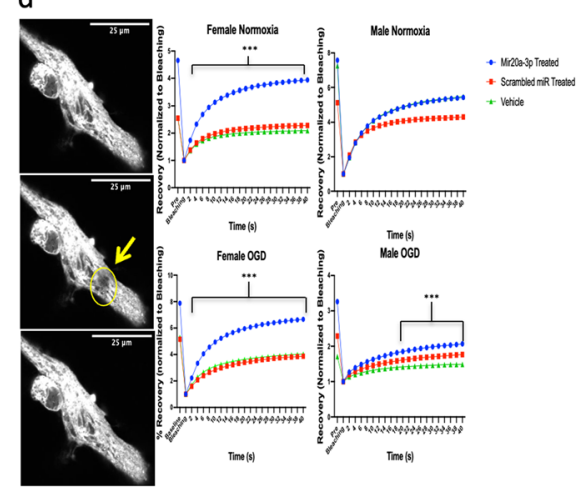

recovery in both OGD and normoxic female groups immediately after bleaching $(<4 \mathrm{~s})$, while the miR-20a-3p - treated male cells only exhibited significantly increased recovery in the OGD group after $18 \mathrm{~s}$ (Fig. 2d). Another functional assay used to assess mitochondrial dynamics is the Mito Stress Test using the Seahorse 96XFe Analyzer. This assay involves the serial injection of various drugs (oligomycin, FCCP, and rotenone + antimycin A) that target components of the electron transport chain. In normoxic conditions, female astrocytes show no difference between the miR-20a-3ptreated group and either of the control groups; however, the miR-20a-3p-treated male astrocytes show reduced oxygen consumption rate relative to both vehicle and scrambled oligo-treated cells (Fig. 2e). Moreover, miR-20a-3p treatment significantly reduces oxygen consumption rates in the male and female astrocytes in OGD conditions, though the male cells exhibit significantly more variability than the female cells (Fig. 2e). Increased oxygen consumption could be indicative of cell senescence induced by oxidative damage [24], and these data suggest that miR-20a-3p may reduce this senescent feature in an ischemic environment.

\section{Astrocyte-Specific Enhancement of MiR-20a-3p Improves Select Stroke Outcomes in Middle-Aged Females}

To assess the neuroprotective potential of astrocytederived miR-20a-3p in the context of stroke, a viral construct (rAAV5-TetOn-GFAP-miR-20a-3p-mCherry, SignaGen) under the control of a TetOn system with a GFAP promoter and conjugated with a mCherry reporter
(Supplementary Figure 3a) and a control vector (Supplementary Figure $3 \mathrm{~b}$ ) was synthesized and injected into the left hemisphere of middle-aged female rat brains. Figure $3 \mathrm{c}$ demonstrates that the recombinant adeno-associated virus (rAAV) is activated by doxycycline and primarily co-localizes with GFAP. Two doses $\left(2.5 \times 10^{11} \mathrm{VP} / \mathrm{ml}\right.$; $2.5 \times 10^{10} \mathrm{VP} / \mathrm{ml}$ ) of the $\mathrm{rAAV}$ were used in pilot studies to determine the most effective dose to assess the effect of astrocyte-specific miR-20a-3p expression after stroke. Striatal injections of the high dose $\left(2.5 \times 10^{11} \mathrm{VP} / \mathrm{ml}\right)$ did not result in any adverse effects on body weight or spleen weight and were analyzed extensively for stroke outcomes (Fig. 3a-e). The Kaplan-Meier survival analysis (Fig. 3a) showed that the striatal injection of the rAAV containing miR-20a-3p reduced stroke-induced mortality $(p=0.0372$ ). Infarct volume (Fig. $3 \mathrm{~b}, \mathrm{c})$ assessed at 5 days after MCAo in animals that received the control vector was greater $(53.42 \%)$ than that in animals that received the miR-20a-3p-containing vector $(40.39 \%)$, although this difference did not meet the criteria for statistical significance $(p=0.1117)$. Sensory motor performance assessed by the adhesive removal test showed that both groups had increased latency at 2 days post stroke on the contralesional limb, while at 5 days post stroke, the miR20a-3p group showed improved recovery and significantly reduced latency as compared to the control vector group (Fig. 3d). The vibrissae-evoked forelimb placement task did not demonstrate any improvement in the percentage of correct responses post MCAo when the same-side vibrissae were stimulated nor when the cross-midline vibrissae were stimulated (Supp. Fig. 4A). 
Fig. 3 Effect of rAAV-TetOnGFAP-miR-20a-3p on stroke outcome in middle-aged females. A Kaplan-Meier survival plot shows significantly greater mortality in animals that received the control vector as compared to the miR-20a-3pcontaining vector. B Representative images of TTC-stained sections obtained 5 days post MCAo. C Histogram depicting mean $( \pm$ SEM $)$ infarct volume. D Histogram of mean $( \pm$ SEM) latency to remove the adhesive tape pre MCAo, 2 days post MCAo, and 5 days post MCAo. $N=12$ (miR-20a-3p) and $n=11$ (scrambled oligo) pre MCAo. $* p \leq 0.05, * * p \leq 0.01$
A
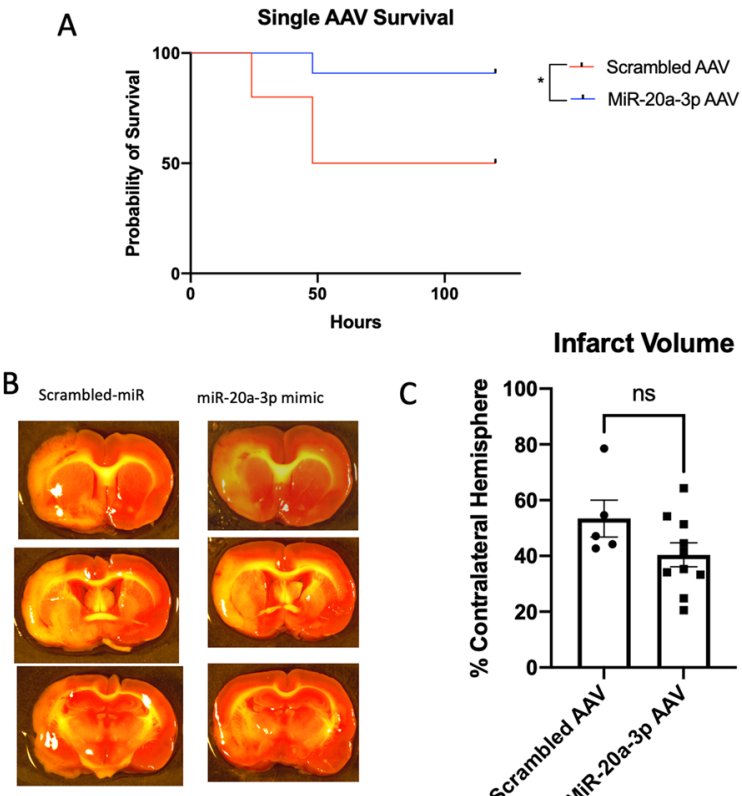

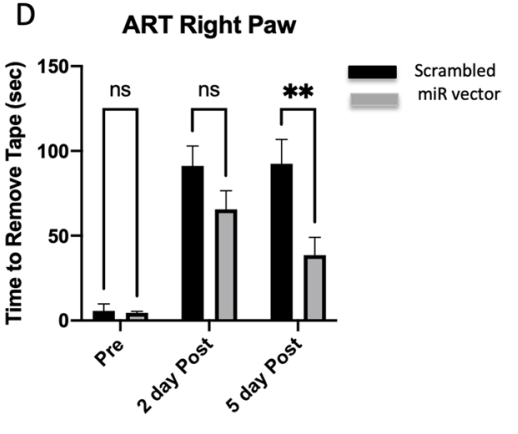

Fig. 4 Cellular localization of i.v. miR-20a-3p. Photomicrographs of coronal sections from animals injected with FAMlabeled miR-20a-3p after MCAo or sham surgery probed for glial and neuronal markers and counterstained for DAPI (blue). A MCAo: GFAP (red). B MCAo: NeuN (red). C Sham: GFAP (red). D Sham: NeuN (red). Arrows indicate FAM-labeled miR-20a-3p (green). E Quantification of FAM-miR-20a-3p co-localization. $* * p \leq 0.01$
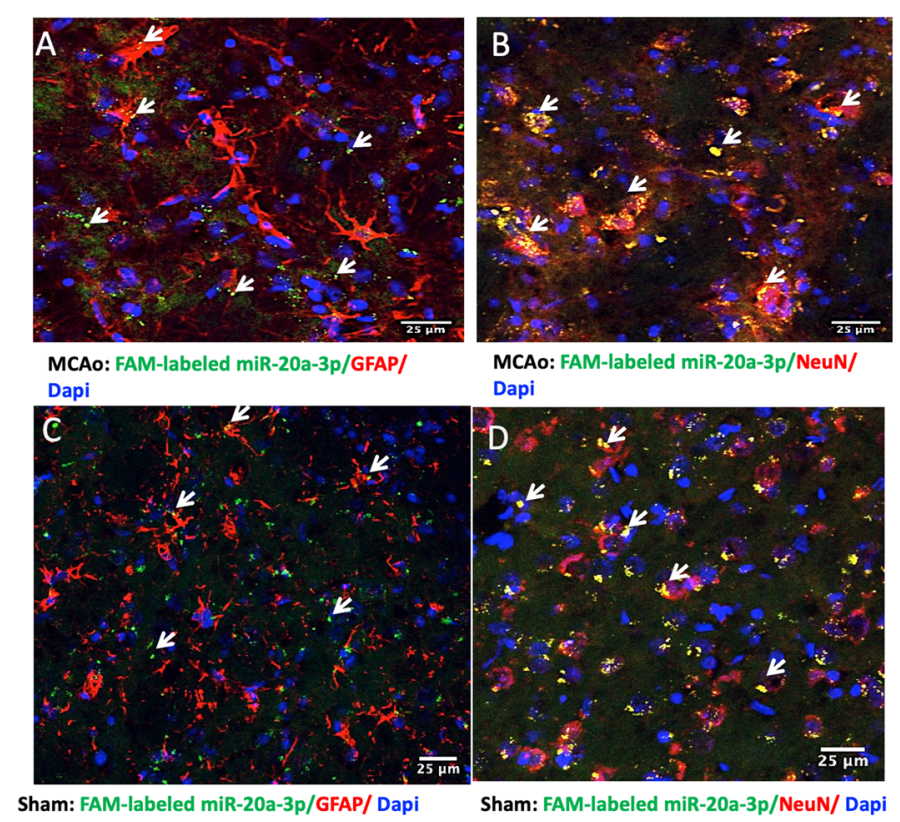

MCAo: FAM-labeled miR-20a-3p/NeuN/ Dapi
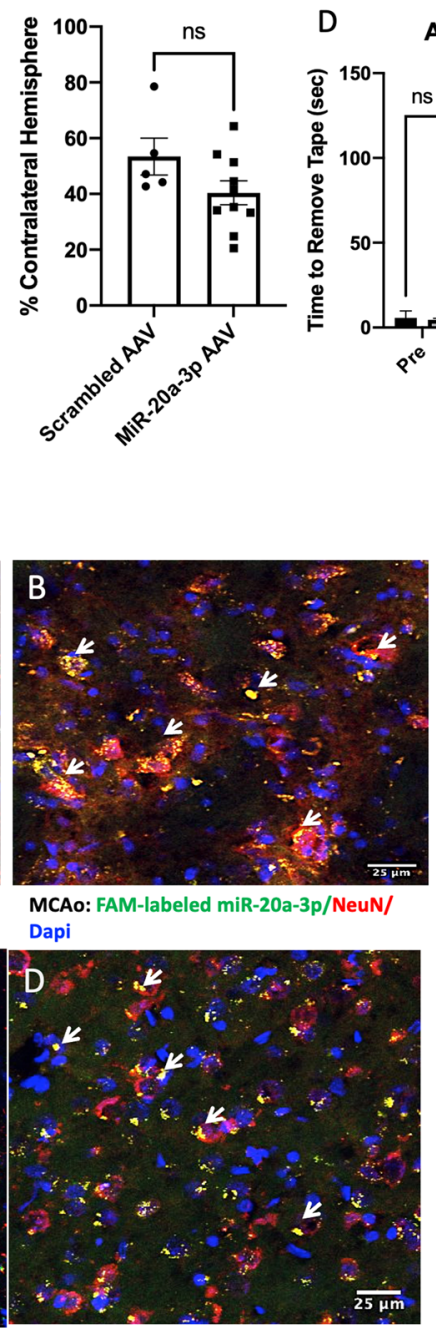

Sham: FAM-labeled miR-20a-3p/NeuN/ Dapi
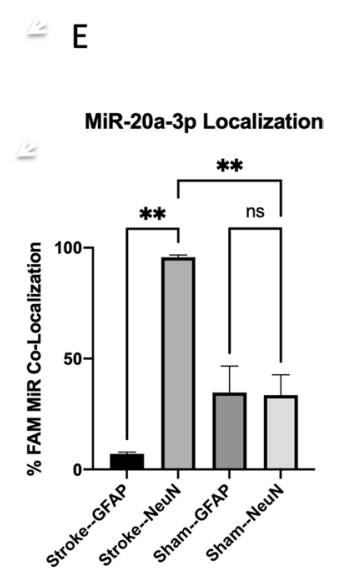

\section{FAM-Labeled miR-20a-3p Localizes to Neurons Rather Than Astrocytes After MCAo}

We next examined the efficacy of intravenously injected miR-20a-3p. First, a FAM-labeled miR-20a-3p mimic was injected via tail vein $4 \mathrm{~h}$ after MCAo or sham surgery. The FAM-labeled mimic was chosen in order to visualize the localization of the miR-20a-3p mimic after intravenous treatment. FAM-labeled miR-20a-3p (green) preferentially co-localized with $\mathrm{NeuN}+$ cells rather than GFAP+ cells in the animals that received MCAo (Fig. 4a, b). The proportion of FAM-labeled miR-20a-3p expressed in cells (co-labeled with dapi) that co-localized with NeuN+ cells compared to GFAP+ cells confirmed that neurons preferentially uptake the FAM-labeled $\mathrm{miR}$ (Fig. 4e). Interestingly, in animals that received the sham surgery, this preferential uptake by neurons is not observed (Fig. 4c-e). These data indicate that neurons may uptake this miRNA more readily under ischemic conditions. 
Fig. 5 Effect of miR20a-3p mimic on neuronal mitochondrial dynamics. a Primary human neurons stained with MitoTracker Deep Red and incubated with FAM-labeled miR-20a-3p for 30 min. b qRTPCR expression of miR-20a-30 in primary human neurons. $\mathbf{c}$ Calcein assay on female and male human neurons. Histogram depicting the mean $( \pm$ SEM) percent of viable cells relative to normoxic conditions. d FRAP analysis of female and male human neurons. The recovery was normalized to the level of bleaching in the cells (bleaching value was set at 1). e Seahorse XFe96 Mito Stress Test on human neurons. $* p \leq 0.05, * * p \leq 0.01$, $* * * p \leq 0.001, * * * * p \leq 0.0001$

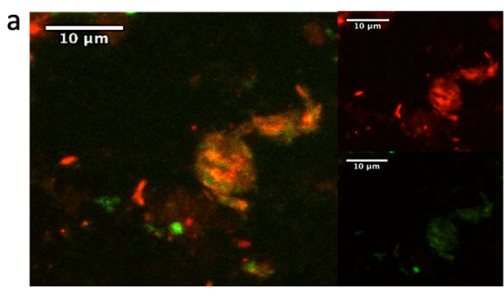

d

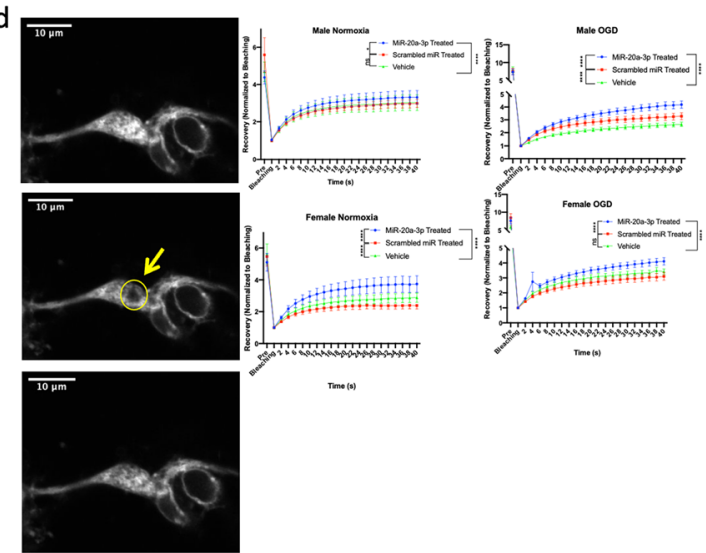

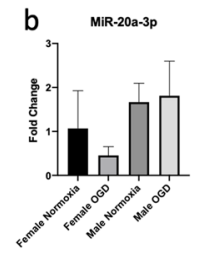
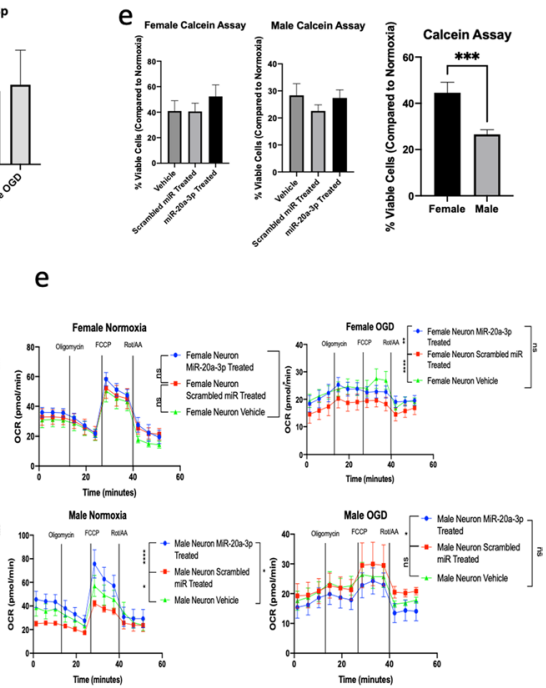

\section{Effect of MiR-20a-3p on Neuronal Mitochondrial Function In Vitro}

In light of the cellular localization of i.v. FAM-miR-20a-3p injections, we decided to investigate the effects of miR20a-3p on neuronal mitochondrial functions. Primary human neurons were cultured in identical conditions to the previously described astrocytes. Neuronal identify was confirmed via immunohistochemistry for NeuN (red) and co-labeled with the nuclear stain DAPI (Supplementary Fig. 2b). OGD was reduced to $30 \mathrm{~min}$ to account for greater cell death of the neurons relative to astrocytes in ischemic conditions. Confocal images indicate that the neurons uptake the FAMlabeled miR-20a-3p mimic after 30 min of OGD (Fig. 5a). qPCR analysis was performed to assess the expression of miR-20a-3p in normoxic and OGD conditions, and no differences in the expression of this microRNA were observed in either sex (Fig. 5b).

A cell viability assay, FRAP analysis, and the Seahorse Mito Stress Test assay were performed to assess the effect of miR-20a-3p on neuronal mitochondrial function. The cell viability assay did not show any improvement of neuronal survival after $30 \mathrm{~min}$ of OGD, though the female neurons did demonstrate a greater degree of viability as compared to the male neurons ( $p=0.0007$, Fig. $5 c$ ); however, key functional differences were elucidated in the FRAP and Seahorse assays. In all cases, neurons treated with miR-20a-3p significantly improved fluorescent recovery after photobleaching (Fig. 5d), similar to what was observed in the astrocytes. The Seahorse assay, however, demonstrated differences between neurons and astrocytes regarding the effect of miR-20a-3p on oxygen consumption. In normoxic conditions, male neurons treated with miR-20a-3p showed significantly elevated levels of oxygen consumption rate relative to control groups (Fig. 5e). The female neurons treated with miR-20a-3p exhibited greater oxygen consumption relative to vehicle, but there was not a significant difference between miR-20a$3 p$-treated and scrambled miR-treated groups. OGD reduced the rate of oxygen consumption in both sexes, though the difference between the miR-20a-3p-treated groups and the vehicle groups is not significant (Fig. 5e).

\section{Neuron-Specific Enhancement of MiR-20a-3p is Neuroprotective in Middle-Aged Female Rats After Stroke}

A second viral construct (rAAV5-TetOn-NSE-miR-20a-3p mCherry, SignaGen) was created to assess the effect of conditional neuronal miR-20a-3p expression. rAAV striatal injections and endothelin-1 (ET-1) surgeries were performed as indicated previously, and all procedures were identical to the previous rAAV experiment. However, unlike the GFAP vector, the neuron-specific enolase (NSE) vector showed significant reduction of infarct volume in animals that received the rAAV vector (Fig. 6b, c, p =0.0087) and more significant improvement in the sensory motor tasks at both 2 days and 5 days post stroke (Fig. 6 d, $p<0.0001$ ). While post-stroke mortality in the rAAV-NSE-miR-20a-3p was almost half that of the group receiving the control vector, the Kaplan-Meier survival analysis only indicated a statistical trend in survival (Fig. 6a, $p=0.0805$ ). 
Fig. 6 Effect of rAAV-TetOnNSE-miR-20a-3p on stroke outcome in middle-aged females. a Kaplan-Meier survival plot shows a trend toward greater mortality in animals that received the control vector as compared to the miR-20a-3pcontaining vector. b Representative images of TTC-stained sections obtained 5 days post MCAo. c Histogram depicting mean $( \pm$ SEM $)$ infarct volume. d Histogram of mean $( \pm$ SEM $)$ latency to remove the adhesive tape pre MCAo, 2 days post MCAo, and 5 days post MCAo. $N=10$ (miR-20a-3p) and $n=9$ (scrambled oligo) pre MCAo. $* p \leq 0.01, * * * * p \leq 0.0001$

Fig. 7 Effect of intravenous miR-20a-3p mimics on stroke outcomes in middle-aged females at IT, DT, and VDT: A(i)-C(i) TTC-stained coronal sections from middle-aged females treated with scrambled oligo or miR-20a-3p. a(ii)-c(ii) Histogram depicts average infarct volume $( \pm$ SEM) normalized to the volume of the non-ischemic hemisphere. a(iii)-c(iii) Sensory motor performance on the adhesive removal test was evaluated before and after stroke. Histograms depict mean $( \pm$ SEM $)$ latency in seconds to remove the tape. DT, $N=6$ (control or treatment); IT, $N=7$ (control) and $N=8$ (treatment); VDT, $N=8$ (control) and $N=8$ (treatment). ${ }^{*} p \leq 0.05, * * p \leq 0.01$

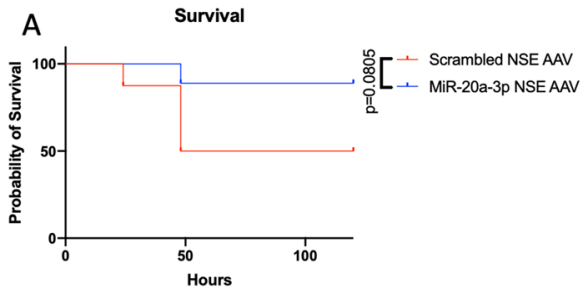

B
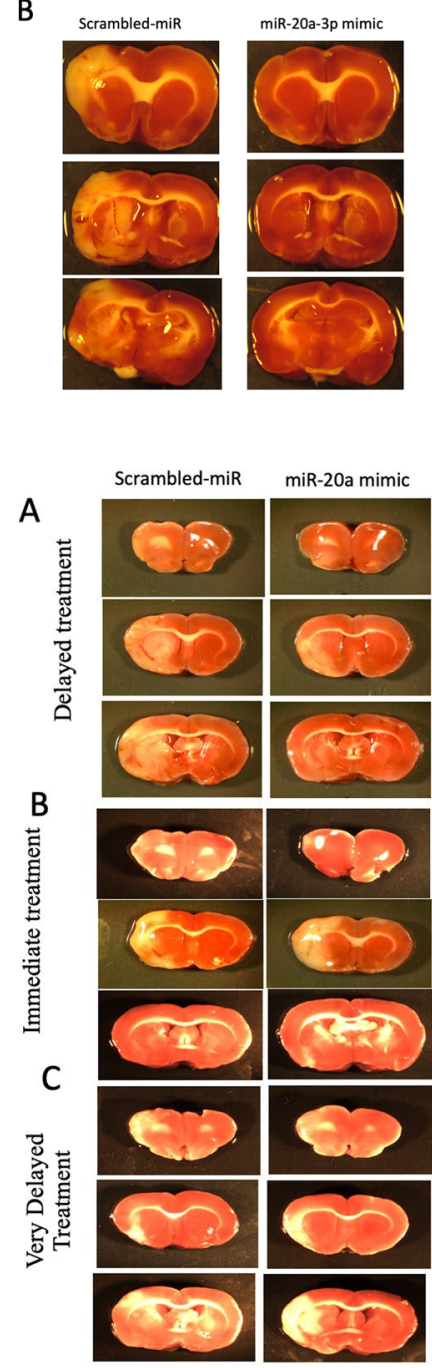

C

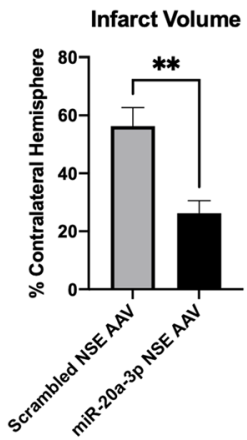

Infarct Volume DT
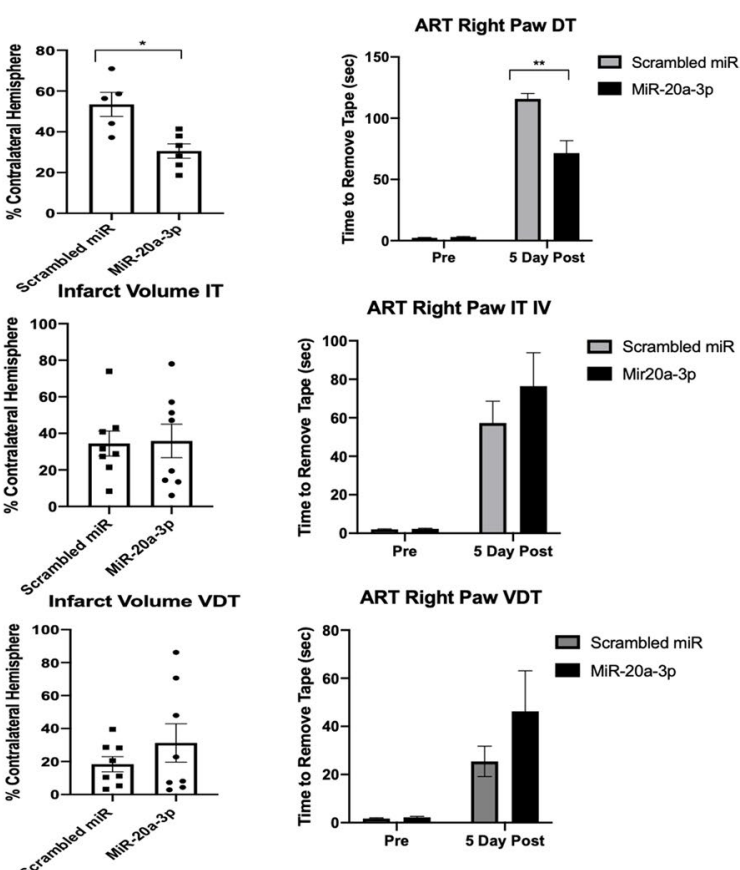

ART Right Paw

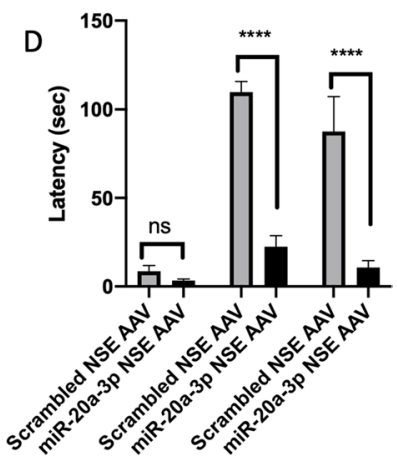

\section{MiR-20a-3p Treatment Significantly Reduced Infarct Volume in Middle-Aged Female and Male Rats}

Although the rAAV experiments provide key insight into the cellular mechanism of neuroprotection of miR$20 a-3 p$, i.v. treatment is much less invasive and therefore more translationally viable, so we next assessed the effect of i.v. miR-20a-3p on acute stroke outcomes. Animals were subjected to MCAo and injected via the tail vein with miR-20a-3p mimic either concurrent (immediate treatment (IT)) with the onset of ischemia, $4 \mathrm{~h}$ after ischemic onset (delayed treatment (DT)), or $24 \mathrm{~h}$ after the onset of ischemia (very delayed treatment (VDT)). As shown in Fig. 7a, triphenyl tetrazolium chloride (TTC)stained images indicate that the cortico-striatal infarct is significantly reduced in middle-aged females in animals that received miR-20-3p $4 \mathrm{~h}$ after MCAo (DT) as compared to the age-matched counterparts that received i.v. injections of the scrambled oligos. Delayed miR-20a-3p treatment decreased infarct volume by $60 \%$ and improved 
performance on the adhesive removal test when measured 5 days post stroke (Fig. 7a). On the other hand, i.v. injections of miR-20a-3p-delivered concurrent with the onset of ischemia (IT) or $24 \mathrm{~h}$ after MCAo (VDT) did not show any decrease in infarct size or reduction in sensory motor deficits (Fig. 7b, c, Supp. Fig. 4c, d).

Middle-aged males (a group that also sustains severe stroke outcomes) showed significantly reduced infarct following delayed miR-20a-3p mimic treatment (Supplementary Fig. 3A, B; $p<0.05$ ) and significantly improved performance on the adhesive removal test, which is impaired after MCAo (Supplementary Fig. 3C). Collectively, these data indicate that, similar to middle-aged female rats, i.v. miR-20a-3p mimic to middle-aged male rats improved stroke outcomes.

\section{Effect of DT MiR-20a-3p Treatment on Gene Targets Post MCAo}

To assess whether i.v. treatment of miR-20a-3p affected astrocyte gene expression, astrocytes were isolated from the ischemic hemisphere for qRT-PCR analysis. In view of expression of MMP-2, MMP-9, and MMP-14 was elevated in the ischemic hemisphere of animals that received scrambled oligo treatment, while this elevation was mitigated in the ischemic hemisphere of animals receiving miR-20a-3p (Fig. $8 \mathrm{a}-\mathrm{c},{ }^{*} p<0.05$ ). MMP activity was assessed by gelatin zymography. Protein was isolated from the ischemic hemisphere of animals subjected to MCAo that received either miR-20a-3p mimic or scrambled oligo (DT). Lytic activity was noted in the region between 50 and $70 \mathrm{kDa}$ (Fig. 8e), which contains MMP-2 and MMP-14. This region was quantified for densitometry by ImageJ and revealed a significant reduction in MMP activity in the miR-20a-3ptreated animals (Fig. 8f). MMPs have been implicated in the blood-brain barrier (BBB) and cerebral microvasculature damage as well as hemorrhagic bleeds [25]. BBB damage was assessed by ELISA for serum GFAP, which is a surrogate measure of BBB leakiness. As shown in Fig. 8g, there was a trend $(p=0.12)$ toward decreased GFAP levels in the miR-20a-3p-treated groups. Hemorrhagic foci were inspected in TTC-stained sections (shown in Fig. 8h) by a code-blinded observer and were found to be present in all $(100 \%)$ scrambled oligo-treated middle-aged female rats and
Fig. 8 Effect of intravenous miR-20a-3p on markers bloodbrain barrier permeability. a-d qPCR analysis of MMPs from the ischemic and non-ischemic hemispheres of scrambled and miR-20a-3p-treated animals. e Representative image of gelatin Zymogram depicting MMP activity from protein lysates from the ischemic hemisphere. f Histogram showing the mean $( \pm)$ of MMP activity from gelatin zymography. g ELISA analysis of GFAP expression in serum. h Representative TTC images of brain slices with hemorrhagic foci from females treated with miR-20a-3p mimic and scrambled oligo. Foci are indicated by white arrows. $N=6$ (miR-20a-3p) and $n=6$ (scrambled oligo). ${ }^{\#} p<0.1,{ }^{*} p \leq 0.05$
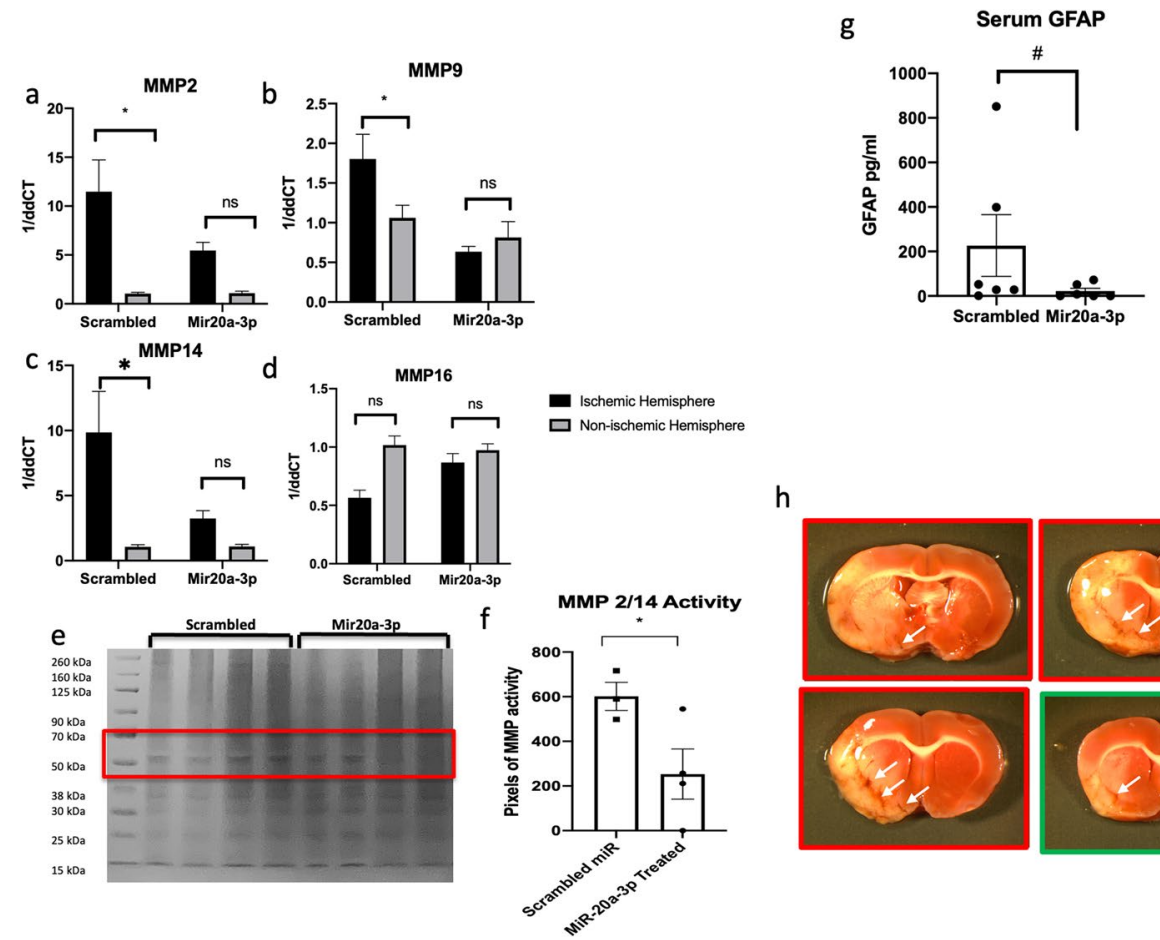

h
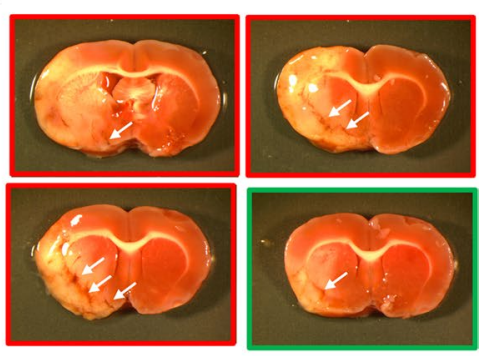

the improved stroke outcomes in the DT group, the analysis was only performed in this group. Several matrix metalloproteinases (MMPs) and mitochondrial gene targets of miR-20a-3p demonstrated altered regulation in the ischemic hemisphere of the animals that received DT as compared to the animal that received scrambled treatment. Gene in one-third of the miR-20a-3p-treated rats (chi-square; $p<0.0143$ ), generally in the vicinity of striatal vessels. Collectively, these data suggest that miR-20a-3p treatment $4 \mathrm{~h}$ after MCAo may protect the brain against MMP-mediated BBB damage and subsequent hemorrhagic transformation. 
Fig. 9 Effect of intravenous miR-20a-3p on predicted mitochondrial targets. a qPCR and b ELISA analysis of IL-17A expression from the ischemic and non-ischemic hemispheres of scrambled and miR-20a-3ptreated animals. $\mathbf{c}-\mathbf{e}$ qPCR analysis of predicted mitochondrial genes from the ischemic and non-ischemic hemispheres of scrambled and miR-20a-3ptreated animals. f Protein from the ischemic hemisphere of scrambled and miR-20a-3ptreated animals probed for Drp1 by western blot and total protein stain. g Histogram of the mean $( \pm$ SEM) of Drp1 normalized to total protein. $N=6(\mathrm{miR}$ 20a-3p) and $n=6$ (scrambled oligo). $* p \leq 0.05, * * p \leq 0.01$
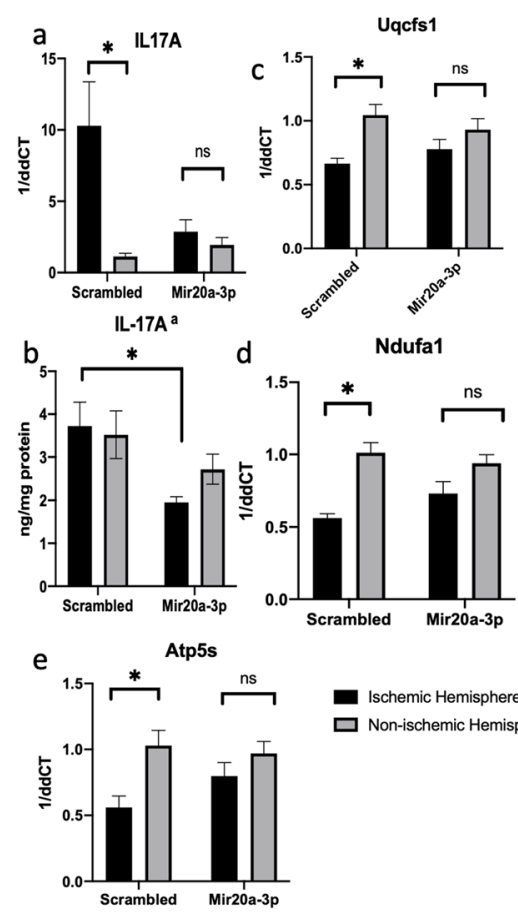
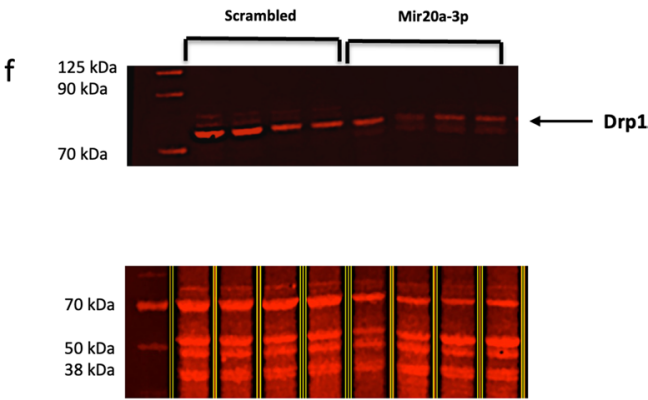

g

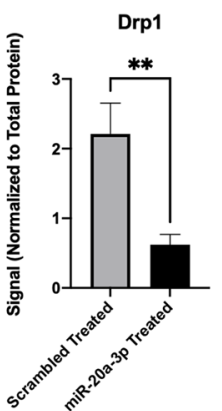

IL-17A is a pro-inflammatory cytokine, which is a predicted target of miR-20a-3p and a therapeutic target for anti-inflammatory drugs to improve recovery post stroke [26]. Similar to the MMPs, IL-17A was elevated in the ischemic hemisphere of animals that received scrambled oligo treatment, while this elevation was mitigated in the ischemic hemisphere of animals receiving miR-20a-3p (Fig. 9a). This was further confirmed by ELISA assay, showing that IL-17A expression was significantly reduced in the ischemic hemisphere of the miR-20a-3p-treated middle-aged females as compared to the scrambled controls (Fig. 9b, $\mathrm{p} \leq 0.05$ ). Circulating (serum) levels of IL-17A were not different in the two groups (data not shown), suggesting that miR-20a-3p either has a brain-specific effect for this cytokine or regulates the blood-brain barrier to prevent extravasation of IL-17A or IL-17A-producing cells.

Mitochondrial gene targets including Uqcfs1, Ndufa1, and Atp5s were reduced in the ischemic hemisphere of the scrambled oligo-treated animals, while there were no differences in the expression of these genes in the two hemispheres of the miR-20a-3p-treated animals (Fig. 9c-e), indicating that miR-20a-3p treatment preserved the expression of mitochondrial genes. Quantitative western blot analysis was performed on protein isolated from the ischemic hemisphere of animals subjected to MCAo that received DT of either miR-20a-3p mimic or scrambled oligo. The blot was probed with a Drp1 (mitochondrial fission protein) antibody (Fig. 9f) and normalized to the LiCOR Total Protein Stain. MiR-20a-3p-treated animals demonstrated significantly less expression of Drp1 as compared to scrambled oligo-treated animals (Fig. 9g).

\section{Discussion}

The present study used well-known age and sex differences in stroke outcomes to identify microRNA with neuroprotective potential. We observed that the miR-17-92 cluster, which has been shown to improve neural plasticity after stroke in male rats/mice [27], was significantly reduced in adult males and middle-aged males and females, groups that typically exhibit poor stroke outcomes, compared to adult females, a group that typically exhibits minimal stroke impairment. Moreover, one member of this miRNA cluster, miR-20a-3p, was dramatically elevated in young females, primarily in astrocytes. In view of the fact that young females sustain smaller infarcts and recover better than other demographics, subsequent studies focused exclusively on this microRNA. These studies used three methods of delivery: astrocyte-specific miR-20a-3p, neuron-specific miR-20a-3p, and i.v. injections of miR-20a-3p. MiR-20a-3p altered mitochondrial function in astrocyte and neuronal cultures, and conditional elevation of this miRNA in astrocytes in middle-aged females partially improved stroke recovery, while conditional expression of miR-20a-3p resulted in robust neuroprotection. Moreover, i.v. injections of miR$20 \mathrm{a}-3 \mathrm{p}$, which is a more tractable therapeutic approach, also improved infarct volume and sensory motor behavior. 
Astrocytes play an important role as neuronal support cells in a variety of ways, a critical one being the maintenance of the BBB [28], which protects the brain's exposure to pathogens and immune cells. Astrocytic end-feet located on the basal lamina surrounding brain microvessels are a critical physical and biochemical barrier. Destruction of this endothelial basal lamina is an early cause of hemorrhage after focal cerebral ischemia [29], resulting from increased activation of MMPs. MMPs, a group of proteolytic zincdependent enzymes, are elevated during stroke in humans [30] and in experimental models of focal ischemia [31] and directly contribute to edema and hemorrhage [32]. Coupled with the loss of endothelial tight junction proteins, elevated levels of MMPs further deteriorate the BBB, augmenting neuroinflammation and cell death. Thus, reduced transcriptional and functional MMP levels may underlie the decreased hemorrhagic foci and robust neuroprotective effects seen in groups treated with miR-20a-3p.

In view of the diverse predicted miR-20a-3p targets, including mitochondrial genes, there may be multiple potential pathways by which miR-20a-3p promotes neuroprotection post stroke. Mitochondrial dysfunction after ischemic stroke is responsible for much of the damage associated with ischemic injury, resulting in massive cell death [33]; thus, drugs that preserve healthy mitochondrial function are critically pursued as therapeutic options. After stroke, the homeostatic balance between mitochondrial fission proteins and mitochondrial fusion proteins is disrupted, resulting in excessive mitochondrial fission, a feature commonly implicated in a number of CNS diseases including Alzheimer's disease [34]. Furthermore, cells subject to ischemia must undergo a transition from utilizing oxygen to facilitate energy production to producing energy via other means such as glycolysis [35]. Data from this study indicated that astrocytes treated with miR-20a-3p showed decreased oxygen consumption relative to other treatment groups, though this reduction is not similarly observed in neurons treated with miR-20a-3p. This may indicate that miR-20a-3p's mechanism of neuroprotection lies in altering metabolic coupling between astrocytes and neurons. This effect in astrocytes may indicate that miR-20a-3p may facilitate effective transition to glycolysis in astrocytes, which may help to facilitate lactate transfer from astrocytes to neurons in ischemic conditions [36]. Alternatively, the reduction in oxygen consumption of the miR-20a-3p-treated astrocytes may simply free up oxygen for other cells such as neurons to consume during ischemic demand. This data supports studies that demonstrate that inhibition of eukaryotic initiation factor 5A hypusination via GC7 treatment, a compound known to reduce basal oxygen consumption, has been shown to be protective after stroke [37] and in an ischemia reperfusion-induced renal injury model [38]. Cellular senescence triggered by oxidative damage has been shown to increase oxygen consumption rate relative to non-senescent cells [24]. Reducing a senescent phenotype in astrocytes may serve to promote neuronal function and survival. Moreover, protein lysates from the brains of rats that were treated with miR-20a-3p after MCAo showed decreased expression of canonical SASP factors, including IL-17A and several MMPs. These data suggest that miR-20a-3p may potentially reduce senescence in these astrocytes, though more studies are needed to confirm this.

In view of their central role in neuronal protection, astrocytic products, including neurosteroids, growth factors, and epigenetic modifiers, are vigorously pursued as stroke therapies. For example, in comparison to any other brain cell, astrocytes produce the most steroids, including progesterone, testosterone, and estradiol, and express enzymes involved in hormone synthesis, including aromatase (Zwain and Yen 1999). An increase in astrocyte-derived estradiol was shown to be neuroprotective and anti-inflammatory following global ischemia, an effect that was blocked by aromatase antisense oligonucleotides (Zhang et al. 2014). Astrocytes also produce multiple growth factors such as VEGF, IGF-1, and BDNF, and decreased expression of these growth factors is a feature of aging astrocytes and a possible explanatory factor for the typically severe stroke outcomes seen in older animals. Our previous work shows that VEGF and IGF-1 are both decreased in the aging astrocyte [22,23]. Additionally, a rAAV-mediated increase in astrocytic IGF-1 reduced infarct volume and stroke-induced sensorimotor deficits in middle-aged female rats [39]. In the case of miRNAs, astrocyte-enriched species such as miR181 and miR29 that target the Bcl-2 family have also been implicated in reducing ischemic cell death $[40,41]$. This study is the first to show that conditional elevation of astrocyte-specific miR-20a-3p improves survival and stroke-induced sensory motor performance, though it had no effect on infarct volume. Moreover, this study also shows that neuron-specific miR-20a-3p was sufficient to significantly improve infarct volume and sensory motor function. The juxtaposition of the fact that astrocytes are the cells that upregulate miR-20a-3p after stroke yet neurons more efficiently utilize the miRNA to improve stroke outcome is interesting. It is possible that this miRNA is specifically transferred from astrocytes to neurons or other neural cell types to confer neuroprotection. Support for this hypothesis comes from a study where extracellular vesicles enriched for the miR-17-92 cluster increase functional recovery after stroke in young male rats [27]. More studies to examine how miR-20a-3p affects neuron-glia interactions are critical to thoroughly understand this interaction.

A unique aspect of this study is the finding that miR$20 a-3 p$ treatment was more effective when administered $4 \mathrm{~h}$ (delayed) after stroke as opposed to immediately after stroke. Studies of stroke neuroprotectants have been criticized for using an unrealistic time window such as pre-stroke 
treatment or treatment that is concurrent with stroke $[42,43]$. The $4 \mathrm{~h}$ (delayed time point) was selected with reference to the outer limit of the therapeutic window for i.v. tPA $(4.5 \mathrm{~h}$ from first symptom) to ensure its translational value, while the $24 \mathrm{~h}$ (very delayed time point) was selected to see if the therapeutic window could be extended. While it was not surprising that the 24-h (very delayed) treatment time point was ineffective, it was surprising to find that concurrent treatment was also not effective. Although the reasons for this are currently not known, we speculate that it may be related to poor uptake of the miRNA by neurons in the early phase of ischemia. Data from this study demonstrates that stroke increases the neuronal uptake of miR-20a-3p as compared to astrocytes (Fig. 5a-e), and one possibility is that it may be due to an increase in the expression of miRNA chaperone proteins. MiRNA chaperones such as the very low-density lipoprotein bind to their membrane receptors (VLDLR) to gain access to cells. Neurons, as do many other cells, increase VLDLR expression after ischemia [44]. Increasing the expression of VLDLR would allow the neuron to more efficiently internalize the microRNA. It is worth noting that VLDLR is also a predicted miR-20a-3p target. It is therefore possible that providing miR-20a-3p too early in the ischemic process reduces the expression of VLDLR resulting in insufficient uptake of the microRNA. Without sufficient uptake, miR-20a-3p is not able to be utilized efficiently and is thus not neuroprotective when given at the onset of the stroke. The 4-h (delayed) time point may be early enough to prevent massive cell death and permanent impairment, yet late enough to allow for sufficient upregulation of VLDLR, thus resulting in a unique, delayed therapeutic window.

Another unusual aspect of these studies is the beneficial effect of miR-20a-3p on both males and females after stroke. As more studies include both sexes in their experimental design, it is clear that drugs are not always equally effective in both sexes. Among the earliest drugs that displayed a sex difference in treatment outcome was the selective pancaspase inhibitor, quinoline-Val-Asp(Ome)-CH2-O-phenoxy (Q-VD-OPh). Female mice exhibit an early release of cytochrome $\mathrm{C}$ and increased caspase activation after stroke, but not males, and that Q-VD-OPh treatment to females decreased infarct volume and improved neurological outcome, but this effect was not observed in male mice [45]. In contrast, PARP inhibitors were effective stroke treatments in male mice and ineffective in females, suggesting that while caspase-dependent cell death pathways are initiated in the ischemic brain of both sexes, males tend to show a preference to PARP- and NO-dependent cell death under ischemic conditions [46, 47]. Recently, we identified miR-363-3p, another stroke-protective miR, using a profiling approach which improved stroke outcomes in females but was found ineffective in males [48]. Interestingly, one of the principal targets of miR-363-3p is caspase-3, a cell death effector, which is consistent with the idea that targeting caspase pathways is therapeutic for females. Sex differences have also been reported for miR-181c, which interacts with the estrogen receptor to improve stroke recovery in females [49]. The unique aspect of the present study is that miR-20a-3p treatment is effective in middle-aged animals of both sexes, which may be linked to its predicted targets such as MMPs and mitochondrial energetics.

These results demonstrate that delayed miR-20a-3p mimic treatment improves acute stroke outcomes and identifies several key pathways by which it may be neuroprotective. However, there are several limitations to this study. Firstly, only one dose of the miRNA was tested. Previous studies from our lab have shown that a single dose of a neuroprotective miRNA is sufficient to improve stroke outcomes [48], though it is entirely possible that multiple doses may further mitigate the severity of damage after stroke. Other potential stroke therapeutics have seen success in adopting a multiple dosage schedule [50-52]. The present study provides justification for further experiments that include more frequent treatment schedules. Another limitation is that this study only assesses the therapeutic potential of miR-20a-3p in the acute phase. Depression and impaired cognitive function are significant long-term symptoms of stroke [53, 54], and improving these long-term consequences is critical for a potential stroke therapeutic. Though mitigating damage in the acute phase may be critical to improving chronic outcomes, future experiments involving chronic behavioral outcomes are necessary to more completely evaluate the therapeutic potential of miR-20a-3p. Finally, we did not assess whether or not these effects of miR-20a-3p are permanent, though there is evidence to suggest that microRNA actions are reversible [55]. Long-term studies involving multiple dosing strategies could shed light on this mechanism.

This study establishes an innovative microRNA-based therapy derived from profiling young and aging astrocytes. Astrocytes are complex cells with the ability to both promote neuronal survival and elevate neuroinflammation, implying both a protective role and a detrimental role for the astrocytes in the brain (reviewed in Ref. [56]). This study also directly compared the conditional expression of miR$20 a-3 p$ in two different cell types, establishing neurons as the primary target for miR-20a-3p-mediated neuroprotection. In view of its sex-independent effects and effective time-delayed treatment option, these studies underscore the potential of miR-20a-3p as a mediator of cell senescence and an effective therapy with translational relevance. 


\section{Methods}

\section{In Vivo Studies}

\section{Animals}

All animals were purchased from Harlan Laboratories (IN). Animals were purchased as adults (6-7 months, 230-320 g) or middle aged (10-12 months, $280-360 \mathrm{~g}$ ). All animals were maintained in a 12-h dark:12-h light cycle in AAALAC-accredited vivarium facilities. Food and water were available ad libitum. A week after arrival, females were smeared daily for 14-21 days to determine estrous status (Jezierski and Sohrabji 2001). Vaginal cells were collected using cotton swabs and placed on slides, and cytology was examined at a low magnification. Adult females with a normal estrous cycle of 4-6 days were included in the study. Middle-aged females were included if cytology indicated they were in constant diestrus for at least 7 consecutive days. Adult animals and middle-aged animals were at an average of 7 months and 11.5 months, respectively, at the time of middle cerebral artery occlusion (MCAo). Within each age and sex, animals were assigned randomly to the treatment groups. A total of 143 animals were used in these studies with group sizes of 5-12. All experimental procedures were conducted in accordance with ARRIVE guidelines [57].

\section{Surgical Procedures}

\section{AAV5 Injections}

Animals were anesthetized (ketamine: $87 \mathrm{mg} / \mathrm{kg}$; xylazine: $13 \mathrm{mg} / \mathrm{kg}$ ) and placed in a stereotaxic instrument for delivery of the adenovirus construct to the cortex and striatum or the striatum alone. The construct was designed as follows: Recombinant adeno-associated virus serotype 5 (AAV5) was packaged (SignaGen, MD) with the miR-20a-3p gene downstream of the astrocyte-specific reporter GFAP and a tetracycline-inducible element (TetOn) and tagged with the mCherry reporter gene. The control construct consisted of an identical shuttle vector without the miR-20a-3p gene. In preliminary studies, the construct was injected at two doses $\left(2.5 \times 10^{11} \mathrm{VP} / \mathrm{ml}\right.$ and $\left.2.5 \times 10^{10} \mathrm{VP} / \mathrm{ml}\right)$ and delivered stereotaxically either to the striatum as a single injection or to the cortex and striatum as two injections. The following coordinates were used relative to bregma on the left hemisphere: for the striatum: $+0.9 \mathrm{~mm}$ anterior/posterior, $+3.6 \mathrm{~mm}$ medial/lateral, and $-6.5 \mathrm{~mm}$ relative to the dura, and for the cortex: $+0.9 \mathrm{~mm}$ anterior/posterior, $+5.5 \mathrm{~mm}$ medial/ lateral, and $-6.0 \mathrm{~mm}$ relative to the dura. Coordinates for the single striatal injections are as follows: $+0.9 \mathrm{~mm}$ anterior/ posterior, $+2.8 \mathrm{~mm}$ medial/lateral, and $-6.5 \mathrm{~mm}$ relative to the dura. In each case, a needle attached to a Hamilton syringe was lowered to the appropriate depth and rAAVTetOn-GFAP-miR-20a-3p-mCherry was delivered slowly into the parenchyma at a rate of $0.5 \mu \mathrm{l} / \mathrm{min}$ for a total of $3.5 \mu 1$. Animals were allowed to recover for 6 weeks after injections to allow full integration of the viral particles prior to MCAo.

\section{Middle Cerebral Artery Occlusion}

All animals were subjected to stereotaxic surgery to occlude the left middle cerebral artery as reported in Refs. [8, 14, 58]. Briefly, MCA occlusion was induced by stereotaxic microinjection of endothelin-1 ( $3 \mu \mathrm{l}, 1: 2$ dilution in DPBS of $1 \mathrm{mg} / \mathrm{ml}$ endothelin-1 stock; American Peptide Company, Inc., CA). ET-1 was injected adjacent to the middle cerebral artery at the following coordinates relative to bregma: $+0.9 \mathrm{~mm}$ anterior/posterior, $+3.4 \mathrm{~mm}$ medial/ lateral, and $-8.5 \mathrm{~mm}$ relative to the dura. For micronome assays, animals were terminated 2 days post stroke. The same procedure was followed for sham surgeries without the injection of endothelin-1. For miR mimic treatment experiments, animals were administered tail vein injections either immediately after stroke (IT), $4 \mathrm{~h}$ post stroke (DT), or $24 \mathrm{~h}$ post stroke (VDT) with $300 \mu \mathrm{l}(7 \mu \mathrm{g} / \mathrm{kg})$ of either miRNA mimic or negative control: miR-20a-3p (ACUGCAUUA CGAGCACUUACA) oligonucleotide sequence (Thermo Fisher, Grand Island, NY) in In Vivo RNA-LANCEr II (Bioo Scientific, Austin, TX). For rAAV experiments, animals were injected i.p. with doxycycline, a stable Tet analogue, to activate the construct. Animals were terminated at 5 days post MCAo for these experiments. At termination, the brain was rapidly removed and processed for TTC staining to assess infarct volume or was collected for RNA and protein extraction. For FAM-miR-20a-3p experiments, animals were injected with a FAM-labeled miR-20a-3p mimic $4 \mathrm{~h}$ after MCAo or sham surgery. These animals were perfused at $48 \mathrm{~h}$ post MCAo. The brains were then extracted and fixed, sucrose loaded, cryosectioned at $30 \mu \mathrm{m}$ per slice, and then mounted on glass slides. The slides were then incubated with the appropriate primary and secondary antibodies (see "Immunohistochemistry") and imaged using a FV12IX83 confocal microscope. The images were then quantified using the following methods: 200 cells (identified by the nuclear dye DAPI) that demonstrated co-localization with the FAM-miR-20a-3p oligo were randomly selected, and the proportion of those cells that were NeuN+ or GFAP+ was quantified.

Infarct Volume Infarct volume estimation was performed on animals terminated on day 5 post stroke using our previously 
described procedures [59]. Briefly, brain slices ( $2 \mathrm{~mm}$ thick) between -2.00 and $+4.00 \mathrm{~mm}$ from bregma were incubated in a $2 \%$ TTC solution at $37{ }^{\circ} \mathrm{C}$ for $20 \mathrm{~min}$ and then photographed using a Nikon E950 digital camera attached to a dissecting microscope. Digitized images were coded and analyzed by an investigator blind to the code. Infarct volume was determined using the Quantity One software package (Bio-Rad, CA) or ImageJ (NIH). Hemorrhagic loci were also visualized in TTC-stained sections by an investigator blind to the code.

\section{Behavioral Assays}

Adhesive Removal Task Sensory motor performance was assessed using procedures described previously for the adhesive removal test $[48,60]$. Briefly, two pieces of adhesive-backed foam tape ( $1 \mathrm{in.} \times 0.5 \mathrm{in}$.) were used as bilateral tactile stimuli attached to the palmar surface of the paw of each forelimb. For each forelimb, the time it took to remove each stimulus (tape) from the forelimbs was recorded during three trials per day for each forepaw. Animals were allowed to rest for $5 \mathrm{~min}$ between sessions, and each test session had a maximum time limit of $120 \mathrm{~s}$.

Vibrissae-Evoked Forelimb Placement Task Stroke injury was assessed using the vibrissae-evoked forelimb placement task, which was performed pre and post MCAo (described by $[59,61])$. Briefly, animals were subjected to same-side placing trials and cross-midline placing trials elicited by stimulating ipsilesional and contralesional vibrissae. Vibrissae-evoked forelimb placement trials revealed a significant loss of right paw placement in all animals post stroke, indicating left-sided cortical-striatal infarction (Supplemental Fig. 4).

Astrocyte microRNA Astrocytes were harvested from the ischemic hemisphere 2 days post stroke, using procedures published in Ref. [23]. Briefly, tissues were dissociated using a neural dissociation kit (trypsin) and cells were passed through a $30-\mu \mathrm{m}$ filter to obtain a single cell suspension. Following myelin removal, cells were collected by positive selection using anti-GLAST antibody (1:5) for $10 \mathrm{~min}$. GLAST was selected as a marker because it is an astrocyte-specific, membrane-associated protein, and previous work has established that astrocytes harvested $48 \mathrm{~h}$ after ischemia express glutamate transporters (GLT-1 and GLAST) and display no age differences in expression.

miRnome Profiling Astrocyte miRnome was assessed in brain astrocytes from the ischemic hemispheres $(n=6$ in each experimental group). Two panels covering 752 mouse and rat miRNAs were used (miRCURY LNA miRNA miRNome PCR Panels).
RNA Extraction RNA was extracted from serum and astrocytes using the miRNeasy Kit (Qiagen, CA) following the manufacturer's instructions, as described in Ref. [14]. Sample purity was assessed by NanoDrop technology, and a ratio of 1.8 was considered acceptable. Samples were stored at $-20{ }^{\circ} \mathrm{C}$ until use.

PCR Amplification Template RNA (25 ng total RNA per sample) was incubated with reverse transcriptase for $60 \mathrm{~min}$ at $42{ }^{\circ} \mathrm{C}$, followed by heat inactivation of the enzyme $(5 \mathrm{~min}$ at $95^{\circ} \mathrm{C}$ ) and used immediately. cDNA was diluted 80-fold and then incubated with SYBR ${ }^{\circledR}$ Green master mix. Ten microliters was dispensed to each tube. An activation/denaturation step $\left(95{ }^{\circ} \mathrm{C}, 10 \mathrm{~min}\right)$ precedes 40 amplification cycles each at $95{ }^{\circ} \mathrm{C}$ for $10 \mathrm{~s}$ and at $60{ }^{\circ} \mathrm{C}$ for $1 \mathrm{~min}$, with a ramp rate of $1.6^{\circ} \mathrm{C} / \mathrm{s}$. MiRNA primers were LNA modified (Exiqon, Woburn, MA) which allows for uniform $\mathrm{T} m$ and confers greater specificity. Samples were then subject to PCR amplification of U6 as a housekeeping gene. Delta CT values of miR-20a-3p were obtained by subtracting the U6 value, and ddCT values were obtained by subtracting the mean $\mathrm{dCT}$ of the adult female group from each value in all groups. The fold change was expressed as the inverse log of $\operatorname{ddCT}\left(1 / 2^{\mathrm{ddCT}}\right)$.

MiRNA expression data obtained from miRnome panels were uploaded into the GSEA (GeneSifter ${ }^{\circledR}$ Analysis Edition) software program (Geospiza). Differences in miRNA expression were identified using a two-way ANOVA using age and sex as two independent factors, with Benjamini and Hochberg correction for false discovery rate for multiple comparisons at a cutoff $(\alpha)$ of 0.05 .

\section{Protein Analysis}

\section{Immunohistochemistry}

Immunofluorescence for NeuN, ALDH1L1, and GFAP were performed on $30-\mu \mathrm{m}$ brain sections mounted on glass slides. The sections were incubated with a blocking buffer ( $5 \%$ bovine serum albumin, $0.1 \%$ Triton $\mathrm{X}-100$ in PBS, $\mathrm{pH}$ 7.4) for $1 \mathrm{~h}$ at room temperature. Sections were then incubated overnight at $4{ }^{\circ} \mathrm{C}$ with primary antibody (NeuN: anti-mouse [EMD Millipore], 1:250 $\mu$; GFAP: anti-rabbit [Sigma-Aldrich], 1:3000 $\mu$ l; Iba1: anti-rabbit [Wako Chemicals], 1:500 $\mu \mathrm{l}$ ). Secondary antibodies (Alexa Fluor 488 and 594 anti-rabbit and anti-mouse) were then used at a 1:500 $\mu \mathrm{l}$ dilution at room temperature for $1 \mathrm{~h}$. The sections were then washed thrice with PBS and then cover slipped with mounting media containing the nuclear dye DAPI (Fluoroshield, Abcam). Sections were visualized and imaged using an FV12-IX83 confocal microscope. 


\section{Protein Extraction}

Cell proteins from the ischemic hemisphere (cortex and striatum) from animals terminated at $48 \mathrm{~h}$ were harvested and lysed in RIPA lysis buffer (Thermo Scientific, Grand Island, NY) and centrifuged at 20,000 rpm for $30 \mathrm{~min}$. Supernatant was collected and stored at $-20{ }^{\circ} \mathrm{C}$ until further analysis. Protein concentrations were determined using the BCA protein assay kit (Pierce, Rockford, IL).

\section{Western Blots}

Protein extract $(30 \mu \mathrm{g})$ from the ischemic hemisphere was loaded into $4-12 \%$ Novex gels and run at $60 \mathrm{~V}$ for $30 \mathrm{~min}$ and then at $100 \mathrm{~V}$ for $90 \mathrm{~min}$. The protein was then transferred onto a PDVF membrane and probed with an antibody for Drp1 (anti-mouse, Abcam, 1:1000 $\mu$ l) and conjugated to a fluorescent secondary antibody (LiCOR goat anti-mouse IRDye 680RD), normalizing to total protein (LiCOR Total Protein Stain).

\section{Zymography}

Protein extract $(25 \mu \mathrm{g})$ from the ischemic hemisphere of middle-aged female rats post MCAo was loaded into a $10 \%$ Novex Zymogram Plus (gelatin) gel and run at $60 \mathrm{~V}$ for $30 \mathrm{~min}$ and then at $100 \mathrm{~V}$ for $90 \mathrm{~min}$ in a non-reducing sample buffer. The gel was then washed and incubated for $24 \mathrm{~h}$ at $37^{\circ} \mathrm{C}$. The gel was then stained with Coomassie blue for $30 \mathrm{~min}$ and then destained until clear bands could be seen.

\section{IL-17a Assay}

IL-17A expression was measured using the Rat IL-17A Platinum ELISA (Thermo Scientific, Grand Island, NY) according to the manufacturer's instructions. Briefly, standards, controls, and aliquots of serum and protein lysates from ischemic cortex and striatum samples were loaded into a 96-well plate pre-coated with antibodies specific for IL-17A and followed by the addition of $100 \mu \mathrm{l}$ of biotin-conjugated anti-rat IL-17A antibody and incubated at room temperature for $2 \mathrm{~h}$ on an orbital microplate shaker at $400 \mathrm{rpm}$ for $30 \mathrm{~s}$. With intervening washes, plates were sequentially incubated with $100 \mu \mathrm{l}$ of streptavidin-HRP for $2 \mathrm{~h}$, and $100 \mu \mathrm{l}$ of TMB substrate solution for $30 \mathrm{~min}$. The color reaction was stopped by an equal volume of stop solution and read at $450 \mathrm{~nm}$ in a microplate reader (Tecan, Switzerland). Standard curves were established from optical densities of wells containing known dilutions of the standard (1.6-100 pg/ml), and sample measurements were interpolated from standard curves. The experiments were performed in duplicates.

\section{In Vitro Studies}

\section{Cell Culture and OGD}

Human astrocytes and neurons from male and female donors of 18-20 gestational weeks of age were purchased from ScienCell Research Laboratories, grown in "Neural Growth Media (NGM)", consisting of Neurobasal media with $2 \%$ B-27 supplement, $2 \%$ heat-inactivated gelded horse serum, $1 \%$ GlutaMAX, $1 \%$ penicillin, $0.1 \%$ ascorbic acid, $0.05 \%$ ampicillin, and $0.05 \%$ kanamycin, and plated in T-25 or T-75 cell culture-treated flasks. For experiments, cells were plated in poly-D-lysine-coated 6- or 96-well plates or in glass-bottom culture dishes in densities appropriate for the assay, which were ascertained via cell titration. Cultures were grown in normoxic conditions $\left(5 \% \mathrm{CO}_{2}\right.$ and $21 \%$ $\mathrm{O}_{2}, 37{ }^{\circ} \mathrm{C}$ ) until confluent. Astrocytes were then subject to OGD $\left(1 \% \mathrm{O}_{2}, 95 \% \mathrm{~N}_{2}\right.$, and $5 \% \mathrm{CO}_{2}$ in glucose-free DMEM, $\left.37^{\circ} \mathrm{C}\right)$ for $6 \mathrm{~h}$ with miR-20a-3p mimic $(50 \mathrm{nM})$, scrambled miRNA $(50 \mathrm{nM})$, or vehicle (PBS). Neurons were subjected to the same OGD conditions for $30 \mathrm{~min}$. Culture media was collected, and cells were used for assays or processed for RNA isolation.

\section{Calcein Assay}

Cells were seeded at a density of $2 \times 10^{4}$ in a 96 -well plate and subjected to OGD and treatment conditions for $6 \mathrm{~h}$. Cell viability was determined using the Calcein-AM dye (Life Technologies, CA). After OGD, cells were incubated with Calcein-AM $(2.5 \mu \mathrm{m})$ in Calcein-AM buffer for $20 \mathrm{~min}$ at $37{ }^{\circ} \mathrm{C}$, and fluorescence was measured on a plate reader (Tecan, Switzerland) with excitation/emission set at $480 \mathrm{~nm}$ and $530 \mathrm{~nm}$, respectively.

\section{FRAP Analysis}

Cells were seeded at a density of $1 \times 10^{5}$ into 2 -ml glass-bottom culture dishes, cultured for 2 days, and then subjected to OGD and treatment conditions for $6 \mathrm{~h}$. Culture media was collected, and cells were washed twice with gas-free recording buffer. Cells were then incubated with $120 \mathrm{nM}$ MitoTracker Deep Red in gas-free recording buffer $(154 \mathrm{mM}$ $\mathrm{NaCl}, 5 \mathrm{mM} \mathrm{KCl}, 2 \mathrm{mM} \mathrm{CaCl} 2 \cdot \mathrm{H}_{2} \mathrm{O}, 0.5 \mathrm{mM} \mathrm{MgCl}{ }_{2} \cdot \mathrm{H}_{2} \mathrm{O}$, $5 \mathrm{mM}$ D-glucose, $10 \mathrm{mM}$ HEPES) for $1 \mathrm{~h}$. After incubation, cells were washed twice with gas-free recording buffer and imaged using confocal microscopy (FV12-IX83). Individual cells with MitoTracker Deep Red labeling were identified, and a region of interest containing a dense area of mitochondria close to the soma was selected. Pre-activation was recorded for $30 \mathrm{~s}$, cells were bleached with high-intensity laser ( $405 \mathrm{~nm}, 80 \%$ power of total laser output) for $3 \mathrm{~s}$, and subsequent activity was recorded for $90 \mathrm{~s}$ post bleach. 
Fluorescence intensity over time was plotted, and cells that did not achieve at least $75 \%$ bleaching were excluded from analysis.

\section{Mitochondrial Respiration}

Cells were seeded at a density of $4 \times 10^{4}$ in 96 -well Seahorse microplates $15 \mathrm{~h}$ before OGD. After OGD, cells were washed twice with Seahorse XF DMEM medium ( $\mathrm{pH}$ 7.4) and incubated for $45 \mathrm{~min}$ in a $\mathrm{CO}_{2}$-free incubator. The Mito Stress Test was then performed according to the manufacturer's instructions. Briefly, this includes taking basal oxygen consumption measurements and then oxygen consumption measurements on the Seahorse XFe96 Analyzer after serial injections of oligomycin, FCCP, and rotenone/antimycin A.

\section{Statistical Analysis}

Power analysis for group sizes was computed based on effect sizes seen in previous data and pilot studies. In order to achieve a power of $0.9(1-\beta)$ and type 1 error rate $(\alpha=0.05)$, the minimum sample size is 5 . For these studies, group sizes ranged from 5 to 10 . For comparisons between adult and middle-aged males and females on miR-20a-3p expression, a two-way ANOVA with post hoc comparisons was performed. For behavioral tests, a two-way ANOVA coded for repeated measure was used for each group, comparing the values obtained pre and post stroke. For all other tests, an unpaired $t$ test was performed. Group differences were considered significant at $p<0.05$ in each case. All in vitro assays were conducted with 3-5 replicate runs, and each run consisted of 3-12 technical replicates. Statistical analysis was performed using unpaired $t$ tests for the Calcein assay, multiple $t$ tests (one per time point) for FRAP assay, and 2-way ANOVA with post hoc comparisons for Seahorse assay. All statistical analyses were performed using GraphPad Prism software (v. 9.0).

Supplementary Information The online version contains supplementary material available at https://doi.org/10.1007/s12975-021-00945-x.

Acknowledgements We would like to thank Dr. Kathiresh Mani, Yumna El-Hakim, Sivani Pandey, Macy Zardeneta, and Crystal Myers for their assistance with the experiments.

Author Contributions TEB, AS, RS, and FS conceived and planned the experiments. TEB, AS, MJP, KEK, and KFK performed the experiments. TEB, AS, MJP, and FS analyzed the results. TEB, AS, RS, and FS wrote the manuscript.

Funding This work was supported by RFAG042189 to FS and 1F31NS118970-01A1 to TEB.
Data Availability This study includes no data deposited in external repositories. Requests for resources and reagents should be directed to the Corresponding Author, Farida Sohrabji (f-sohrabji@tamu.edu).

Code Availability Not applicable.

\section{Declarations}

Conflict of interest The authors declare no competing interests.

Ethical Approval All experiments involving animals were reviewed and approved by the Texas A\&M University Institutional Animal Care and Use Committee in accordance with OLAW guidelines for the humane treatment of animals in research.

Open Access This article is licensed under a Creative Commons Attribution 4.0 International License, which permits use, sharing, adaptation, distribution and reproduction in any medium or format, as long as you give appropriate credit to the original author(s) and the source, provide a link to the Creative Commons licence, and indicate if changes were made. The images or other third party material in this article are included in the article's Creative Commons licence, unless indicated otherwise in a credit line to the material. If material is not included in the article's Creative Commons licence and your intended use is not permitted by statutory regulation or exceeds the permitted use, you will need to obtain permission directly from the copyright holder. To view a copy of this licence, visit http://creativecommons.org/licenses/by/4.0/.

\section{References}

1. De Los Ríos La Rosa FK, Kissela J, Flaherty BM, Alwell ML, Moomaw K, Khatri CJ, Adeoye P, Woo O, Ferioli D, Kleindorfer S. Eligibility for intravenous recombinant tissue-type plasminogen activator within a population: the effect of the European Cooperative Acute Stroke Study (ECASS) III Trial. Stroke. 2012;43(6):1591-5. https://doi.org/10.1161/STROK EAHA.111.645986.

2. Schwamm LHA, Reeves SF, Smith MJ, Saver EE, Messe JL, Bhatt S, Grau-Sepulveda DL, Peterson MV, Fonarow ED. Temporal trends in patient characteristics and treatment with intravenous thrombolysis among acute ischemic stroke patients at Get with the Guidelines-Stroke Hospitals. Circulation. 2013;6(5):543-9. https://doi.org/10.1161/CIRCOUTCOMES. 111.000095.

3. Iorio MV, et al. MicroRNA gene expression deregulation in human breast cancer. Can Res. 2005;65(16):7065-70.

4. Randall $\mathrm{G}$, et al. Cellular cofactors affecting hepatitis C virus infection and replication. Proc Natl Acad Sci USA. 2007;104(31):12884-9.

5. Keller A, et al. Multiple sclerosis: microRNA expression profiles accurately differentiate patients with relapsing-remitting disease from healthy controls. PLoS ONE. 2009;4(10):e7440.

6. Martins M, et al. Convergence of miRNA expression profiling, $\alpha$-synuclein interacton and GWAS in Parkinson's disease. PLoS ONE. 2011;6(10):e25443.

7. Ambros V. MicroRNAs: tiny regulators with great potential. Cell. 2001;107(7):823-6.

8. Selvamani A, Sathyan P, Miranda RC, Sohrabji F. An antagomir to microRNA Let7f promotes neuroprotection in an ischemic stroke model. PLoS ONE. 2012;7(2):e32662. https://doi.org/ 10.1371/journal.pone.0032662. 
9. Harraz MM, Eacker SM, Wang X, Dawson TM, Dawson VL. MicroRNA-223 is neuroprotective by targeting glutamate receptors. Proc Natl Acad Sci USA. 2012;109(46):18962-7.

10. Liu DZ, et al. Elevating microRNA-122 in blood improves outcomes after temporary middle cerebral artery occlusion in rats. J Cereb Blood Flow Metab. 2016;36(8):1374-83.

11. Panta A, Montgomery K, Nicolas M, Mani KK, Sampath D, Sohrabji F. Mir363-3p treatment attenuates long-term cognitive deficits precipitated by an ischemic stroke in middle-aged female rats. Front Aging Neurosci. 2020;12:310. https://doi.org/ 10.3389/fnagi.2020.586362.

12. Panta A, Pandey S, Duncan IN, Duhamel S, Sohrabji F. Mir363$3 p$ attenuates post-stroke depressive-like behaviors in middleaged female rats. Brain Behav Immun. 2019. https://doi.org/10. 1016/j.bbi.2019.01.003.

13. Sohrabji F, Selvamani A. Sex differences in miRNA as therapies for ischemic stroke. Neurochem Int. 2018. https://doi.org/10. 1016/j.neuint.2018.10.021.

14. Selvamani A, Williams MH, Miranda RC, Sohrabji F. Circulating miRNA profiles provide a biomarker for severity of stroke outcomes associated with age and sex in a rat model. Clin Sci. 2014;127(2):77-89. https://doi.org/10.1042/cs20130565.

15. Liu ZC. M, Astrocytes, therapeutic targets for neuroprotection and neurorestoration in ischemic stroke. Prog Neurobiol. 2016;144:103-20. https://doi.org/10.1016/j.pneurobio.2015.09. 008.

16. Goodall EF, et al. Age-associated changes in the blood-brain barrier: comparative studies in human and mouse. Neuropathol Appl Neurobiol. 2018;44(3):328-40.

17. Maciel-Barón LÁ, et al. The secretory phenotype of senescent astrocytes isolated from Wistar newborn rats changes with anti-inflammatory drugs, but does not have a short-term effect on neuronal mitochondrial potential. Biogerontology. 2018;19(5):415-33.

18. Bhat R, et al. Astrocyte senescence as a component of Alzheimer's disease. PLoS ONE. 2012;7(9):e45069.

19. Campuzano O, Castillo-Ruiz M, Acarin L, Castellano B, Gonzalez B. Increased levels of proinflammatory cytokines in the aged rat brain attenuate injury-induced cytokine response after excitotoxic damage. J Neurosci Res. 2009;87(11):2484-97.

20. Lye JJ, et al. Astrocyte senescence may drive alterations in GFAP $\alpha$, CDKN2A p14 ARF, and TAU3 transcript expression and contribute to cognitive decline. Geroscience. 2019;41(5):561-73.

21. Li M, et al. Astrocyte-derived interleukin-15 exacerbates ischemic brain injury via propagation of cellular immunity. Proc Natl Acad Sci USA. 2017;114(3):E396-405.

22. Lewis DK, Thomas KT, Selvamani A, Sohrabji F. Age-related severity of focal ischemia in female rats is associated with impaired astrocyte function. Neurobiol Aging. 2012;33(6):1123.

23. Chisholm NC, et al. Histone methylation patterns in astrocytes are influenced by age following ischemia. Epigenetics. 2015;10(2):142-52.

24. Kim S-J, et al. Mitochondrial peptides modulate mitochondrial function during cellular senescence. Aging (Albany NY). 2018;10(6):1239-56. https://doi.org/10.18632/aging.101463.

25. Turner RJ, Sharp FR. Implications of MMP9 for blood brain barrier disruption and hemorrhagic transformation following ischemic stroke. Front Cell Neurosci. 2016;10:56.

26. Swardfager W, Winer DA, Herrmann N, Winer S, Lanctôt KL. Interleukin-17 in post-stroke neurodegeneration. Neurosci Biobehav Rev. 2013;37(3):436-47.

27. Xin $\mathrm{H}$, et al. MicroRNA-17-92 cluster in exosomes enhance neuroplasticity and functional recovery after stroke in rats. Stroke. 2017;48(3):747-53.
28. Kimelberg HK, Nedergaard M. Functions of astrocytes and their potential as therapeutic targets. Neurotherapeutics. 2010;7(4):338-53.

29. Hamann GF, Okada Y, del Zoppo GJ. Hemorrhagic transformation and microvascular integrity during focal cerebral ischemia/ reperfusion. J Cereb Blood Flow Metab. 1996;16(6):1373-8.

30. Clark AW, Krekoski CA, Bou S-S, Chapman KR, Edwards DR. Increased gelatinase A (MMP-2) and gelatinase B (MMP-9) activities in human brain after focal ischemia. Neurosci Lett. 1997;238(1-2):53-6.

31. Romanic AM, Madri JA. Extracellular matrix-degrading proteinases in the nervous system. Brain Pathol. 1994;4(2):145-56.

32. Rosenberg GA, Mun-Bryce S, Wesley M, Kornfeld M. Collagenase-induced intracerebral hemorrhage in rats. Stroke. 1990;21(5):801-7.

33. He Z, Ning N, Zhou Q, Khoshnam SE, Farzaneh M. Mitochondria as a therapeutic target for ischemic stroke. Free Radical Biol Med. 2020;146:45-58.

34. Grohm J, et al. Inhibition of Drp1 provides neuroprotection in vitro and in vivo. Cell Death Differ. 2012;19(9):1446-58.

35. Ham PB III, Raju R. Mitochondrial function in hypoxic ischemic injury and influence of aging. Prog Neurobiol. 2017;157:92-116.

36. Mazucanti CH, Kawamoto EM, Mattson MP, Scavone C, Camandola S. Activity-dependent neuronal Klotho enhances astrocytic aerobic glycolysis. J Cereb Blood Flow Metab. 2019;39(8):1544-56.

37. Bourourou $\mathrm{M}$, et al. Inhibition of eIF5A hypusination pathway as a new pharmacological target for stroke therapy. J Cereb Blood Flow Metab. 2021;41(5):1080-90.

38. Melis N, et al. Targeting eIF5A hypusination prevents anoxic cell death through mitochondrial silencing and improves kidney transplant outcome. J Am Soc Nephrol. 2017;28(3):811-22.

39. Okoreeh AK, Bake S, Sohrabji F. Astrocyte-specific insulin-like growth factor-1 gene transfer in aging female rats improves stroke outcomes. Glia. 2017;65(7):1043-58.

40. Ouyang L, et al. Programmed cell death pathways in cancer: a review of apoptosis, autophagy and programmed necrosis. Cell Prolif. 2012;45(6):487-98.

41. Ouyang Y-B, Giffard RG. MicroRNAs affect BCL-2 family proteins in the setting of cerebral ischemia. Neurochem Int. 2014;77:2-8.

42. Gladstone DJ, Black SE, Hakim AM. Toward wisdom from failure: lessons from neuroprotective stroke trials and new therapeutic directions. Stroke. 2002;33(8):2123-36.

43. Grotta JC. Acute stroke therapy at the millennium: consummating the marriage between the laboratory and bedside: the Feinberg Lecture. Stroke. 1999;30(8):1722-8.

44. Cal R, et al. Low-density lipoprotein receptor-related protein 1 mediates hypoxia-induced very low density lipoprotein-cholesteryl ester uptake and accumulation in cardiomyocytes. Cardiovasc Res. 2012;94(3):469-79.

45. Liu F, Li Z, Li J, Siegel C, Yuan R, McCullough LD. Sex differences in caspase activation after stroke. Stroke. 2009;40(5):1842-8.

46. Siegel C, Turtzo C, McCullough LD. Sex differences in cerebral ischemia: possible molecular mechanisms. J Neurosci Res. 2010;88(13):2765-74. https://doi.org/10.1002/jnr.22406.

47. Gibson CL, Attwood L. The impact of gender on stroke pathology and treatment. Neurosci Biobehav Rev. 2015. https://doi.org/10. 1016/j.neubiorev.2015.08.020.

48. Selvamani A, Sohrabji F. Mir363-3p improves ischemic stroke outcomes in female but not male rats. Neurochem Int. 2017;107:168-81.

49. Stary CM, et al. Inhibition of miR-181a protects female mice from transient focal cerebral ischemia by targeting astrocyte estrogen 
receptor- $\alpha$. Mol Cell Neurosci. 2017;82:118-25. https://doi.org/ 10.1016/j.mcn.2017.05.004.

50. Chen Y-J, Raman G, Bodendiek S, O'Donnell ME, Wulff H. The KCa3.1 blocker TRAM-34 reduces infarction and neurological deficit in a rat model of ischemia/reperfusion stroke. J Cerebr Blood Flow Metab. 2011;31(12):2363-74.

51. Zhang D, et al. ENT1 inhibition attenuates apoptosis by activation of cAMP/pCREB/Bcl2 pathway after MCAO in rats. Exp Neurol. 2020;331:113362.

52. Nazari M, Keshavarz S, Rafati A, Namavar MR, Haghani M. Fingolimod (FTY720) improves hippocampal synaptic plasticity and memory deficit in rats following focal cerebral ischemia. Brain Res Bull. 2016;124:95-102.

53. Panta A, Montgomery K, Nicolas M, Mani KK, Sampath D, Sohrabji F. Mir363-3p treatment attenuates long-term cognitive deficits precipitated by an ischemic stroke in middle-aged female rats. Front Aging Neurosci. 2020;12:1.

54. Panta A, Pandey S, Duncan IN, Duhamel S, Sohrabji F. Mir363-3p attenuates post-stroke depressive-like behaviors in middle-aged female rats. Brain Behav Immun. 2019;78:31-40.

55. Bhattacharyya SN, Habermacher R, Martine U, Closs EI, Filipowicz W. Relief of microRNA-mediated translational repression in human cells subjected to stress. Cell. 2006;125(6):1111-24.

56. Chisholm NC, Sohrabji F. Astrocytic response to cerebral ischemia is influenced by sex differences and impaired by aging. Neurobiol Dis. 2016;85:245-53.
57. Kilkenny C, Browne WJ, Cuthill IC, Emerson M, Altman DG. Improving bioscience research reporting: the ARRIVE guidelines for reporting animal research. PLoS Biol. 2010;8(6):e1000412.

58. Selvamani A, Sohrabji F. The neurotoxic effects of estrogen on ischemic stroke in older female rats is associated with age-dependent loss of insulin-like growth factor-1. J Neurosci. 2010;30(20):6852-61.

59. Selvamani A, Sohrabji F. Reproductive age modulates the impact of focal ischemia on the forebrain as well as the effects of estrogen treatment in female rats. Neurobiol Aging. 2010;31(9):1618-28.

60. Balden R, Selvamani A, Sohrabji F. Vitamin D deficiency exacerbates experimental stroke injury and dysregulates ischemia-induced inflammation in adult rats. Endocrinology. 2012;153(5):2420-35.

61. Woodlee MT, Asseo-García AM, Zhao X, Liu S-J, Jones TA, Schallert T. Testing forelimb placing "across the midline" reveals distinct, lesion-dependent patterns of recovery in rats. Exp Neurol. 2005;191(2):310-7.

Publisher's Note Springer Nature remains neutral with regard to jurisdictional claims in published maps and institutional affiliations. 